\title{
Biosynthetic gene clusters, secondary metabolite profiles, and cards of virulence in the
}

\section{closest nonpathogenic relatives of Aspergillus fumigatus}

4 Jacob L. Steenwyk ${ }^{1}$, Matthew E. Mead ${ }^{1,+}$, Sonja L. Knowles ${ }^{2,+}$, Huzefa A. Raja ${ }^{2}$, Christopher D.

5 Roberts $^{2}$, Oliver Bader ${ }^{3}$, Jos Houbraken ${ }^{4}$, Gustavo H. Goldman ${ }^{5}$, Nicholas H. Oberlies ${ }^{2}$, Antonis

$8{ }^{1}$ Department of Biological Sciences, Vanderbilt University, Nashville, TN, 37235, USA

$9{ }^{2}$ Department of Chemistry and Biochemistry, University of North Carolina at Greensboro,

10 Greensboro, NC, USA

$11{ }^{3}$ Institute for Medical Microbiology, University Medical Center Göttingen, Göttingen, Germany

$12{ }^{4}$ Westerdijk Fungal Biodiversity Institute, Uppsalalaan 8, 3584 CT Utrecht, the Netherlands

$13{ }^{5}$ Faculdade de Ciências Farmacêuticas de Ribeirão Preto, Universidade de São Paulo, São Paulo,

\section{Brazil}

$15+$ M. E. M. and S. L. K. contributed equally to this work.

16

$17{ }^{*}$ Correspondence: antonis.rokas@vanderbilt.edu

19 Running title: Secondary metabolism in A. fumigatus and its relatives

21 Keywords: secondary metabolites, specialized metabolism, gliotoxin, chemodiversity,

22 mycotoxin, biosynthetic gene cluster, cards of virulence, pathogenicity, 


\section{Abstract}

24 Aspergillus fumigatus is a major human pathogen that causes hundreds of thousands of infections

25 yearly with high mortality rates. In contrast, Aspergillus fischeri and the recently described

26 Aspergillus oerlinghausenensis, the two species most closely related to A. fumigatus, are not

27 known to be pathogenic. Some of the "cards of virulence" that A. fumigatus possesses are

28 secondary metabolites that impair the host immune system, protect from host immune cell

29 attacks, or acquire key nutrients. Secondary metabolites and the biosynthetic gene clusters

30 (BGCs) that typically encode them often vary within and between fungal species. To gain insight

31 into whether secondary metabolism-associated cards of virulence vary between $A$. fumigatus, $A$.

32 oerlinghausenensis, and A. fischeri, we conducted extensive genomic and secondary metabolite

33 profiling analyses. By analyzing multiple A. fumigatus, one A. oerlinghausenensis, and multiple

34 A. fischeri strains, we identified both conserved and diverged secondary metabolism-associated

35 cards of virulence. For example, we found that all species and strains examined biosynthesized

36 the major virulence factor gliotoxin, consistent with the conservation of the gliotoxin BGC

37 across genomes. However, species differed in their biosynthesis of fumagillin and pseurotin,

38 both contributors to host tissue damage during invasive aspergillosis; these differences were

39 reflected in sequence divergence of the intertwined fumagillin/pseurotin BGCs across genomes.

40 These results delineate the similarities and differences in secondary metabolism-associated cards

41 of virulence between a major fungal pathogen and its nonpathogenic closest relatives, shedding

42 light into the genetic and phenotypic changes associated with the evolution of fungal

43 pathogenicity. 


\section{$44 \quad$ Importance}

45 The major fungal pathogen Aspergillus fumigatus kills tens of thousands each year. In contrast,

46 the two closest relatives of A. fumigatus, namely Aspergillus fischeri and Aspergillus

47 oerlinghausenensis, are not considered pathogenic. A. fumigatus virulence stems, partly, from its

48 ability to produce small molecules called secondary metabolites that have potent activities during

49 infection. In this study, we examined whether A. fumigatus secondary metabolites and the

50 metabolic pathways involved in their production are conserved in A. oerlinghausenensis and $A$.

51 fischeri. We found that the nonpathogenic close relatives of $A$. fumigatus produce some, but not

52 all, secondary metabolites thought to contribute to the success of $A$. fumigatus in causing human

53 disease and that these similarities and differences were reflected in the underlying metabolic

54 pathways involved in their biosynthesis. Compared to its nonpathogenic close relatives, $A$.

55 fumigatus produces a distinct cocktail of secondary metabolites, which likely contributes to these

56 organisms' vastly different potentials to cause human disease. More broadly, the study of

57 nonpathogenic organisms that have virulence-related traits, but are not currently considered

58 agents of human disease, may facilitate the prediction of species capable of posing future threats

59 to human health. 
$\underline{\text { Introduction }}$

61 Fungal diseases impose a clinical, economic, and social burden on humans (Drgona et al., 2014;

62 Vallabhaneni et al., 2016; Benedict et al., 2019). Fungi from the genus Aspergillus are

63 responsible for a considerable fraction of this burden, accounting for more than 250,000

64 infections annually with high mortality rates (Bongomin et al., 2017). Aspergillus infections

65 often result in pulmonary and invasive diseases that are collectively termed aspergillosis. Among

66 Aspergillus species, Aspergillus fumigatus is the primary etiological agent of aspergillosis (Latgé

67 and Chamilos, 2019).

69 Even though A. fumigatus is a major pathogen, its closest relatives are not considered pathogenic

70 (Mead et al., 2019a; Steenwyk et al., 2019; Rokas et al., 2020a). Numerous studies have

71 identified factors that contribute to A. fumigatus pathogenicity, such as the organism's ability to

72 grow well at higher temperatures and in hypoxic conditions (Kamei and Watanabe, 2005; Tekaia

73 and Latgé, 2005; Abad et al., 2010; Grahl et al., 2012). Factors that contribute to pathogenicity

74 could be conceived as analogous to individual "cards" of a "hand" (set of cards) in a card game -

75 that is, individual factors are typically insufficient to cause disease but can collectively do so

76 (Casadevall, 2007).

78 Several secondary metabolites biosynthesized by $A$. fumigatus are known "cards" of virulence

79 because of their involvement in impairing the host immune system, protecting the fungus from

80 host immune cell attacks, or acquiring key nutrients (Raffa and Keller, 2019). For example, the

81 secondary metabolite gliotoxin has been shown to contribute to A. fumigatus virulence by

82 inhibiting the host immune response (Sugui et al., 2007). Other secondary metabolites implicated 
83 in virulence include: fumitremorgin, which inhibits the activity of the breast cancer resistance

84 protein (González-Lobato et al., 2010); verruculogen, which modulates the electrophysical

85 properties of human nasal epithelial cells (Khoufache et al., 2007); trypacidin, which is cytotoxic

86 to lung cells (Gauthier et al., 2012); pseurotin, which inhibits immunoglobulin E (Ishikawa et al.,

87 2009); and fumagillin which causes epithelial cell damage (Guruceaga et al., 2018) and impairs

88 the function of neutrophils (Fallon et al., 2010, 2011) (Table 1).

90 By extension, the metabolic pathways responsible for the biosynthesis of secondary metabolites

91 are also "cards" of virulence. Genes in these pathways are typically organized in contiguous sets

92 termed biosynthetic gene clusters (BGCs) (Keller, 2019). BGCs are known to evolve rapidly, and

93 their composition can differ substantially across species and strains (Lind et al., 2015, 2017;

94 Rokas et al., 2018, 2020b). For example, even though $A$. fumigatus contains 33 BGCs and $A$.

95 fischeri contains 48 BGCs, only 10 of those BGCs appear to be shared between the two species

96 (Mead et al., 2019a). Interestingly, one of the BGCs that is conserved between A. fumigatus and

97 A. fischeri is the gliotoxin BGC and both species have been shown to biosynthesize the toxic

98 virulence factor (Knowles et al., 2020). These results suggest that the gliotoxin "card" is part of a

99 winning "hand" that facilitates virulence only in the background of the major pathogen $A$.

100 fumigatus and not in that of the nonpathogen $A$. fischeri (Knowles et al., 2020).

101

102 To date, such comparisons of BGCs and secondary metabolite profiles among A. fumigatus and

103 closely related nonpathogenic species have been few and restricted to single strains (Mead et al.,

104 2019a; Knowles et al., 2020). However, genetic and phenotypic heterogeneity among strains of a

105 single species has been shown be an important factor when studying Aspergillus pathogenicity 
(Kowalski et al., 2016, 2019; Keller, 2017; Ries et al., 2019; Bastos et al., 2020; Santos et al.,

107

108

109 trypacidin, whose effects include broad suppression of the immune response system and lung cell

2020). Examination of multiple strains of A. fumigatus and close relatives - including the recently described and largely uncharacterized (in the context of pathogenicity) closest known relative of $A$. fumigatus, $A$. oerlinghausenensis (Houbraken et al., 2016)—will increase our understanding of the A. fumigatus secondary metabolite "cards" of virulence.

To gain insight into the genomic and chemical similarities and differences in secondary metabolism among $A$. fumigatus and nonpathogenic close relatives, we characterized variation in BGCs and secondary metabolites produced by A. fumigatus and nonpathogenic close relatives.

To do so, we first sequenced and assembled $A$. oerlinghausenensis $\mathrm{CBS} 139183^{\mathrm{T}}$ as well as $A$. fischeri strains NRRL 4585 and NRRL 4161 and analyzed them together with four $A$. fumigatus and three additional $A$. fischeri publicly available genomes. We also characterized the secondary metabolite profiles of three A. fumigatus, one $A$. oerlinghausenensis, and three $A$. fischeri strains. On the one hand, we found that the biosynthesis of the secondary metabolites gliotoxin and fumitremorgin, which are both known to interact with mammalian cells (Yamada et al., 2000; González-Lobato et al., 2010; Li et al., 2012; Raffa and Keller, 2019), as well as their BGCs, were conserved among pathogenic and nonpathogenic strains. Interestingly, we found only $A$. fischeri strains, but not A. fumigatus strains, biosynthesized verruculogen, which changes the electrophysical properties of human nasal epithelial cells (Khoufache et al., 2007). On the other hand, we found that both A. fumigatus and A. oerlinghausenensis biosynthesized fumagillin and damage (Ishikawa et al., 2009; Fallon et al., 2010, 2011; Gauthier et al., 2012), but A. fischeri did not. These results reveal that nonpathogenic close relatives of $A$. fumigatus also produce some, 
129 but not all, of the secondary metabolism-associated cards of virulence known in A. fumigatus.

130 Further investigation of the similarities and differences among A. fumigatus and close

131 nonpathogenic relatives may provide additional insight into the "hand of cards" that enabled $A$.

132 fumigatus to evolve into a deadly pathogen.

\section{$134 \quad \underline{\text { Results }}$}

\section{Conservation and diversity of biosynthetic gene clusters within and between species}

136 We sequenced and assembled $A$. oerlinghausenensis CBS $139183^{\mathrm{T}}$ and $A$. fischeri strains NRRL

1374585 and NRRL 4161. Together with publicly available genomes, we analyzed 10 Aspergillus

138 genomes (five A. fischeri strains; four A. fumigatus strains; one A. oerlinghausenensis strain; see

139 Methods). We found that the newly added genomes were of similar quality to other publicly

140 available draft genomes (average percent presence of BUSCO genes: $98.80 \pm 0.10 \%$; average

$141 \quad$ N50: 451,294.67 \pm 9,696.11; Fig. S1). We predicted that $A$. oerlinghausenensis CBS $139183^{\mathrm{T}}, A$.

142 fischeri NRRL 4585, and A. fischeri NRRL 4161 have 10,044, 11,152 and 10,940 genes,

143 respectively, numbers similar to publicly available genomes. Lastly, we inferred the evolutionary

144 history of the 10 Aspergillus genomes using a concatenated matrix of 3,041 genes $(5,602,272$

145 sites) and recapitulated species-level relationships as previously reported (Houbraken et al.,

146 2016). Relaxed molecular clock analyses suggested that $A$. oerlinghausenensis CBS $139183^{\mathrm{T}}$

147 diverged from $A$. fumigatus approximately $3.9(6.4-1.3)$ million years ago and that $A$.

148 oerlinghausenensis and A. fumigatus split from $A$. fischeri approximately 4.5 (6.8 - 1.7) million

149 years ago (Fig. 1A; Fig. S2). 
151 Examination of the total number of predicted BGCs revealed that $A$. fischeri has the largest BGC

152 repertoire. Among A. fumigatus, A. oerlinghausenensis, and A. fischeri, we predicted $50.80 \pm$

$1532.17,40,35.75 \pm 2.22$ BGCs, respectively, and found they spanned diverse biosynthetic classes

154 (e.g., polyketides, non-ribosomal peptides, terpenes, etc.) (Fig. 1B). Network-based clustering of

155 BGCs into cluster families (or groups of homologous BGCs) resulted in qualitatively similar

156 networks when we used moderate similarity thresholds (or edge cut-off values; Fig. S3). Using a

157 (moderate) similarity threshold of 0.5 , we inferred 88 cluster families of putatively homologous

158 BGCs (Fig. 1C).

160 Examination of BGCs revealed extensive presence and absence polymorphisms within and

161 between species. We identified 17 BGCs that were present in all 10 Aspergillus genomes

162 including the hexadehydroastechrome (HAS) BGC (cluster family 311 or CF311), the

163 neosartoricin BGC (CF61), and other putative BGCs likely encoding unknown products (Fig.

164 S4A; Table S1). In contrast, we identified 18 BGCs found in single strains, which likely encode

165 unknown products. Between species, similar patterns of broadly present and species-specific

166 BGCs were observed. For example, we identified 18 BGCs that were present in at least one

167 strain across all species; in contrast, A. fumigatus, A. oerlinghausenensis, and A. fischeri had 16,

168 eight, and 27 BGCs present in at least one strain but absent from the other species, respectively.

169 These results suggest each species has a largely distinct repertoire of BGCs. 


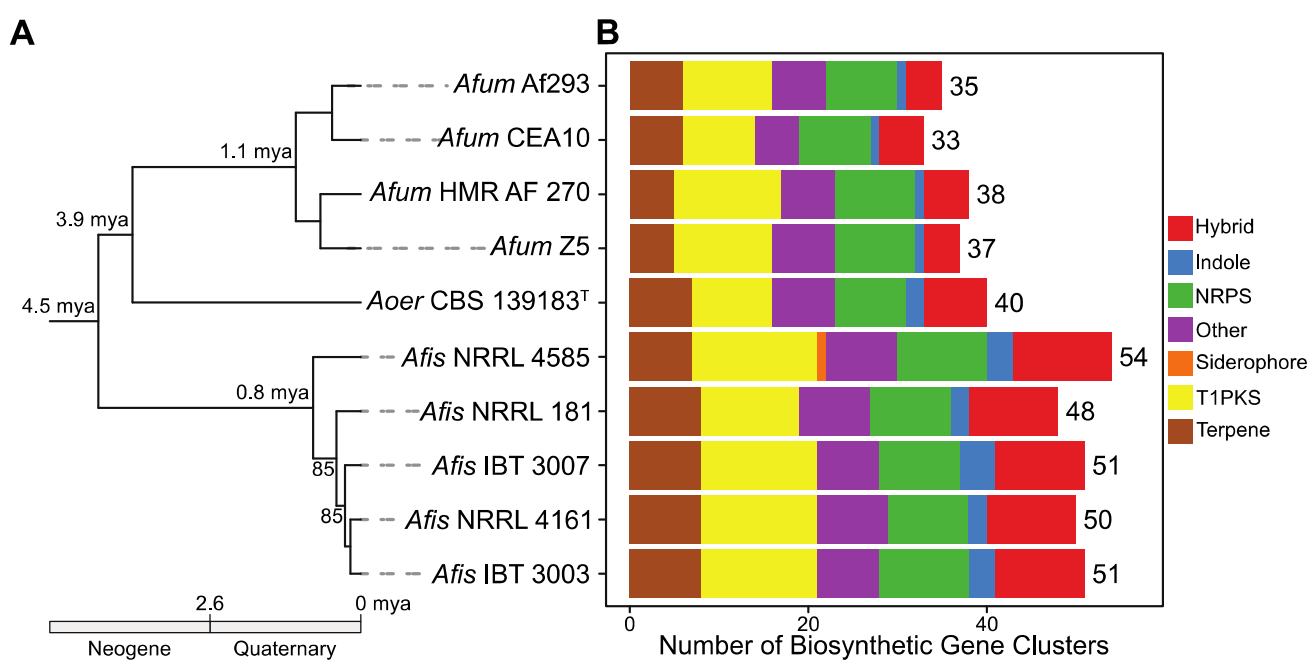

C

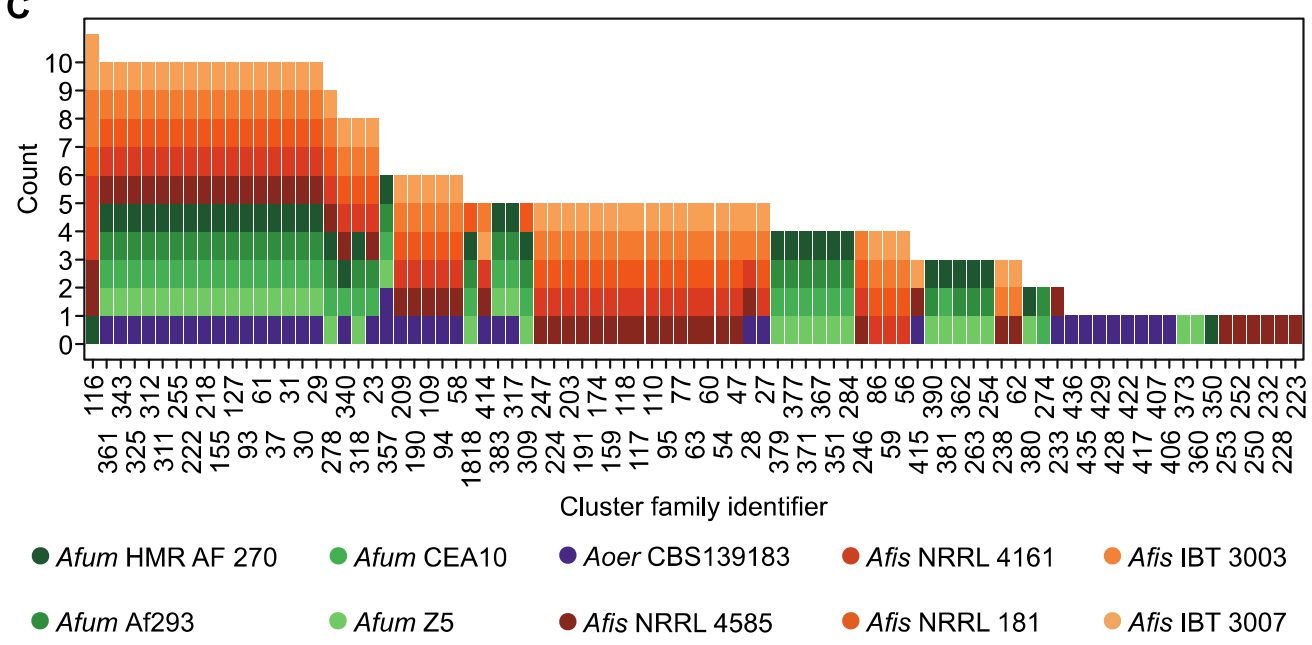

173 Figure 1. Diverse genetic repertoire of biosynthetic gene clusters and extensive presence and

174 absence polymorphisms between and within species. (A) Genome-scale phylogenomic confirms $A$.

175 oerlinghausenensis is the closest relative to $A$. fumigatus. Relaxed molecular clock analyses suggest $A$.

176 fumigatus, $A$. oerlinghausenensis, and A. fischeri diverged from one another during the Neogene geologic

177 period. Bipartition support is depicted for internodes that did not have full support. (B) A. fumigatus

178 harbors the lowest number of BGCs compared to its two closest relatives. (C) Network-based clustering

179 of BGCs into cluster families reveal extensive cluster presence and absence polymorphisms between

180 species and strains. Cluster family identifiers are depicted on the x-axis; the number of strains

181 represented in a cluster family are shown on the y-axis; the colors refer to a single strain from each

182 species. Genus and species names are written using the following abbreviations: Afum: A. fumigatus; 
186 Examination of shared BGCs across species revealed $A$. oerlinghausenensis $\mathrm{CBS} 139183^{\mathrm{T}}$ and $A$.

187 fischeri shared more BGCs with each other than either did with A. fumigatus. Surprisingly, we

188 found ten homologous BGCs between $A$. oerlinghausenensis CBS $139183^{\mathrm{T}}$ and $A$. fischeri but

189 only three homologous BGCs shared between A. fumigatus and A. oerlinghausenensis CBS

$190139183^{\mathrm{T}}$ (Fig. 2A; Fig. S4B) even though $A$. oerlinghausenensis is more closely related to $A$.

191 fumigatus than to $A$. fischeri (Fig. 1A). BGCs shared by A. oerlinghausenensis CBS $139183^{\mathrm{T}}$ and

192 A. fischeri were uncharacterized while BGCs present in both $A$. fumigatus and $A$.

193 oerlinghausenensis CBS $139183^{\mathrm{T}}$ included those that encode fumigaclavine and

194 fumagillin/pseurotin. Lastly, to associate each BGC with a secondary metabolite in A. fumigatus

195 Af293, we cross referenced our list with a publicly available one (Table S2). Importantly, all

196 known A. fumigatus Af293 BGCs were represented in our analyses.

198 More broadly, examination of shared and species-specific gene families revealed that $A$.

199 oerlinghausenensis does not have many species-specific gene families and shares more gene

200 families with $A$. fischeri than $A$. fumigatus (Fig. 2B). Specifically, we noted that $A$.

201 oerlinghausenensis CBS $139183^{\mathrm{T}}$ has only eight species-specific gene families; in contrast, $A$.

202 fischeri and A. fumigatus have 1,487 and 548 species-specific gene families, respectively. 
A

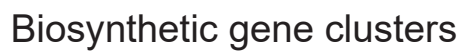

B
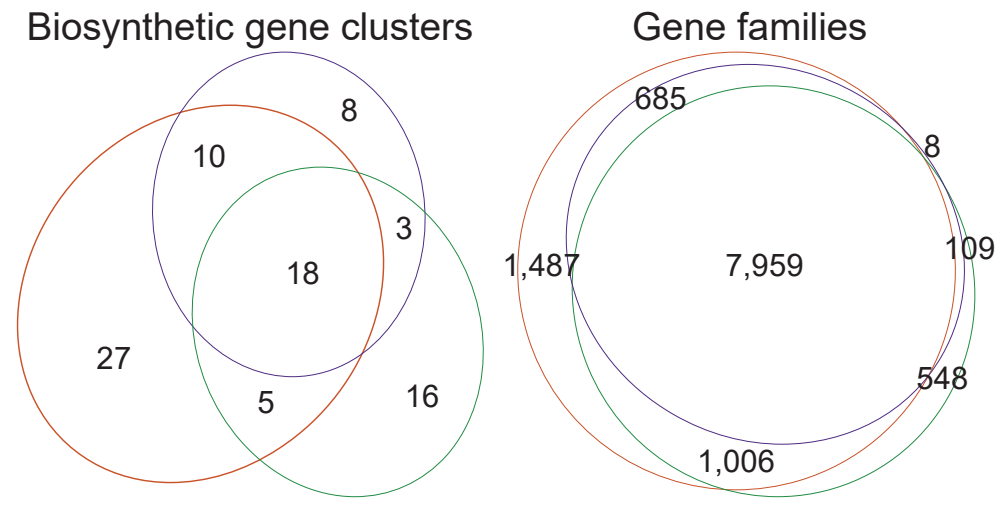

Aspergillus fischeri
Aspergillus oerlinghausenensis

Aspergillus fumigatus

205 Figure 2. Aspergillus oerlinghausenensis shares more gene families and BGCs with A. fischeri

206 than A. fumigatus. (A) Euler diagram showing species-level shared BGCs. (B) Euler diagram showing

207 species-level shared gene families. In both diagrams, $A$. oerlinghausenensis shares more gene families

208 or BGCs with A. fischeri than A. fumigatus despite a closer evolutionary relationship.

210 Despite a closer evolutionary relationship between A. oerlinghausenensis and A. fumigatus, we

211 found $A$. oerlinghausenensis shares more gene families with $A$. fischeri compared to $A$. fumigatus

212 (685 and 109, respectively) suggestive of extensive gene loss in the A. fumigatus stem lineage.

213 Lastly, we observed strain heterogeneity in gene family presence and absence within both $A$.

214 fumigatus and A. fischeri (Fig. S5).

216 Within and between species variation in secondary metabolite profiles of $\boldsymbol{A}$. fumigatus and

\section{7 its closest relatives}

218 To gain insight into variation in secondary metabolite profiles within and between species, we

219 profiled A. fumigatus strains Af293, CEA10, and CEA17 (a pyrG1/URA3 derivative of CEA10),

220 A. fischeri strains NRRL 181, NRRL 4585, and NRRL 4161, and A. oerlinghausenensis CBS

$221139183^{\mathrm{T}}$ for secondary metabolites. Specifically, we used three different procedures, including 
222 the isolation and structure elucidation of metabolites, where possible, followed by two different

223 metabolite profiling procedures that use mass spectrometry techniques. Altogether, we isolated

224 and characterized 19 secondary metabolites; seven from A. fumigatus, two from $A$.

225 oerlinghausenensis, and ten from A. fischeri (Fig. S6). These products encompassed a wide

226 diversity of secondary metabolite classes, such as those derived from polyketide synthases, non-

227 ribosomal peptide-synthetases, terpene synthases and mixed biosynthesis enzymes.

229 To characterize the secondary metabolites biosynthesized that were not produced in high enough

230 quantity for structural identification through traditional isolation methods, we employed

231 “dereplication" mass spectrometry protocols specific to natural products research on all tested

232 strains at both $30^{\circ} \mathrm{C}$ and $37^{\circ} \mathrm{C}$ (see supporting information, dereplication example; figshare:

233 10.6084/m9.figshare.12055503) (El-Elimat et al., 2013; Ito and Masubuchi, 2014; Gaudêncio

234 and Pereira, 2015; Hubert et al., 2017). We found an overlap of secondary metabolites between

235 strains of the same species (Table S3); for example, monomethylsulochrin (3) was isolated from

236 A. fumigatus Af293, but through metabolite profiling, its spectral features were noted also in $A$.

237 fumigatus strains CEA10 and CEA17. We identified metabolites that were biosynthesized by

238 only one species; for example, pseurotin A (4) was solely present in A. fumigatus strains.

239 Furthermore, we also found an overlap of several secondary metabolites across species, such as

240 fumagillin (6), which was biosynthesized by $A$. fumigatus and $A$. oerlinghausenensis, and

241 fumitremorgin B (16), which was biosynthesized by strains of both $A$. oerlinghausenensis and $A$.

242 fischeri. Together, these analyses suggest that closely related Aspergillus species and strains

243 exhibit variation both within as well as between species in the secondary metabolites produced. 
245 To further facilitate comparisons of secondary metabolite profiles within and between species, 246 we used the 1,920 features (i.e., unique $\mathrm{m} / \mathrm{z}$ - retention time pairs) that were identified from all 247 strains at all temperatures (Fig. 3A), to perform Principal Components Analysis (PCA)

A

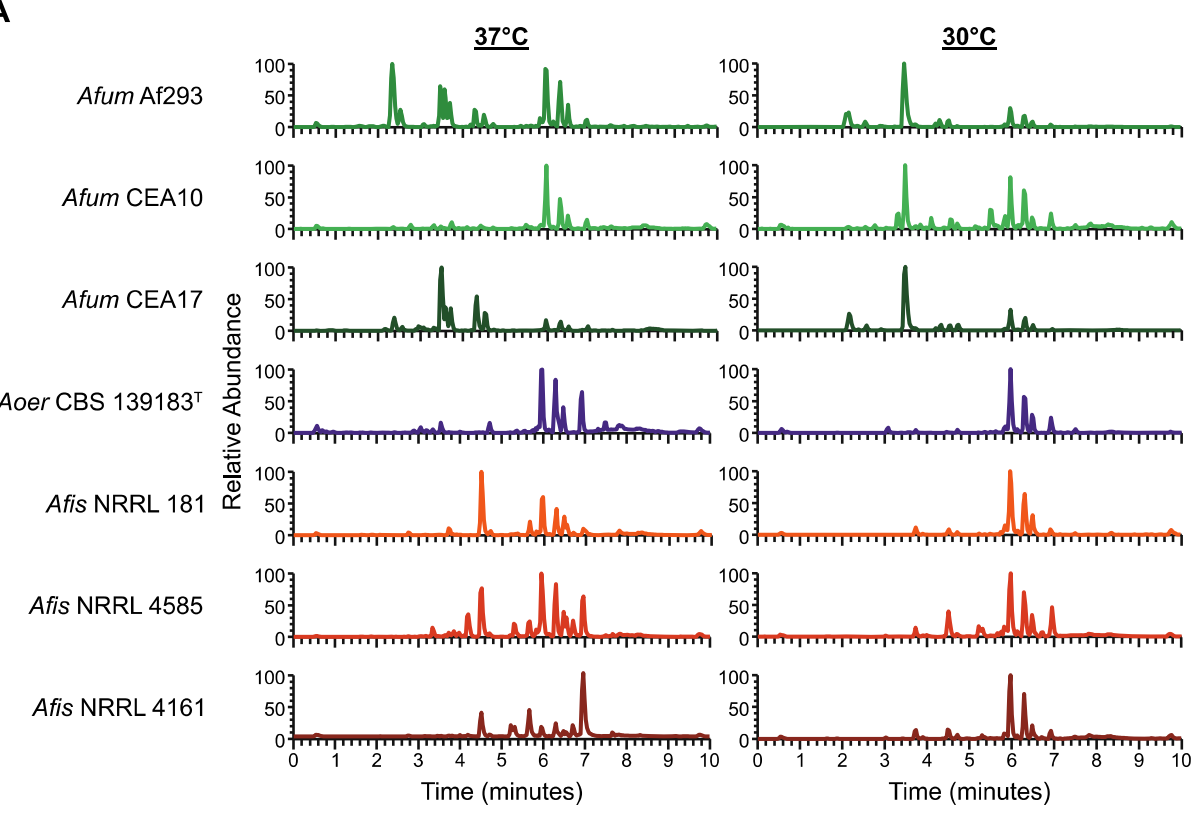

B

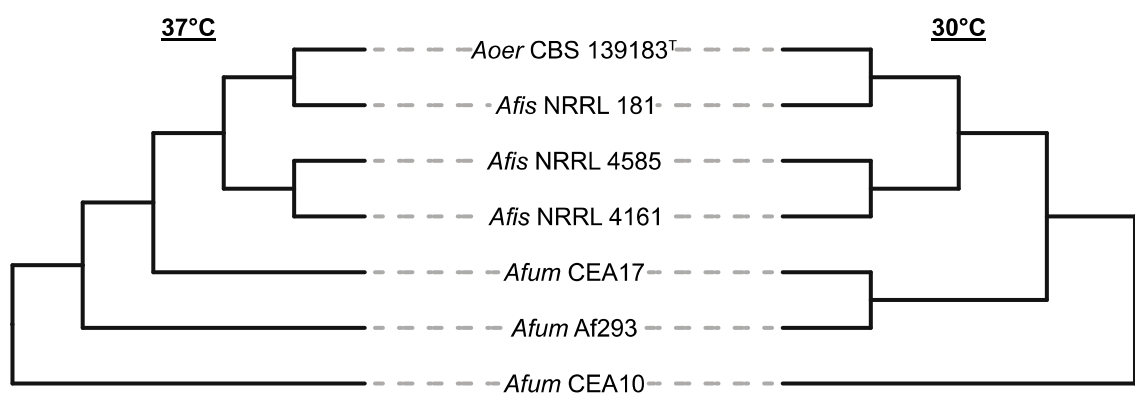

Figure 3. A. oerlinghausenensis and $A$. fischeri have more similar secondary metabolite profiles

251 than $A$. fumigatus. (A) UPLC-MS chromatograms of secondary metabolite profiles of $A$. fumigatus and

252 its closest relatives, $A$. oerlinghausenensis and $A$. fischeri at $37^{\circ} \mathrm{C}$ and $30^{\circ} \mathrm{C}$ (left and right, respectively).

253 (B) Hierarchical clustering of chromatograms reveals $A$. oerlinghausenensis clusters with $A$. fischeri and 254 not its closest relative, A. fumigatus at $37^{\circ} \mathrm{C}$ and $30^{\circ} \mathrm{C}$ (left and right, respectively). 
chromatograms at both $37^{\circ} \mathrm{C}$ and $30^{\circ} \mathrm{C}$ (Fig. 3B, S7B, and S7C) indicated that the three $A$.

258 fischeri strains and A. oerlinghausenensis were the most chemically similar to each other. When

259 we generated PCA plots from only the compounds that could be isolated from each culture (44

260 features that represent the 19 isolated compounds) at both $37^{\circ} \mathrm{C}$ (Fig. S7D) and $30^{\circ} \mathrm{C}$ (Fig. S7E),

261 the chemical similarities between A. oerlinghausenensis and A. fischeri were even more evident,

262 with this clustering showing similar results to the total feature dendrogram (Fig. 3B). These data

263 suggest that there are more similarities among the secondary metabolites that are biosynthesized

264 in higher abundance. While the clustering of $A$. oerlinghausenensis CBS $139183^{\mathrm{T}}$ and $A$. fischeri

265 NRRL 181 is conserved, these strains were also shown to be similar to A. fumigatus CEA10 (Fig.

266 S7D-E). These combined results suggest that at lower temperatures, such as $30^{\circ} \mathrm{C}$, there is a more

267 varied response in how BGCs are being utilized, leading to a more diverse production of

268 chemical compounds.

270 In summary, our chemical analyses suggest that the secondary metabolite profiles of $A$.

271 oerlinghausenensis and A. fischeri are more similar to each other than to A. fumigatus (Fig. 3B

272 and S7B-E). This finding is surprising because phylogenetic analysis indicates that $A$.

273 oerlinghausenensis is more closely related to A. fumigatus than it is to A. fischeri (Fig. 1A).

274 However, the similarity of secondary metabolite profiles of $A$. oerlinghausenensis and $A$. fischeri

275 is consistent with our finding that the genome of $A$. oerlinghausenensis shares higher numbers of

276 BGCs and gene families with $A$. fischeri than with A. fumigatus (Fig. 2). Similarly, clustering

277 patterns in secondary metabolite-based plots (Fig. S7B-E) resemble those of BGC-based plots

278 (Fig. S7A), suggesting that the observed similarities in the chemotypes of $A$. oerlinghausenensis

279 and $A$. fischeri are broadly reflected in their metabolism-associated genotypes. 


\section{Conservation and divergence among biosynthetic gene clusters implicated in $\boldsymbol{A}$. fumigatus}

\section{2 pathogenicity}

283 Secondary metabolites are known to play a role in A. fumigatus virulence (Raffa and Keller,

284 2019). We therefore conducted a focused examination of specific A. fumigatus BGCs and

285 secondary metabolites that have been previously implicated in the organism's ability to cause

286 human disease (Table 1). We found varying degrees of conservation and divergence that were

287 associated with the absence or presence of a secondary metabolite. Among conserved BGCs that

288 were also associated with conserved secondary metabolite production, we highlight the

289 mycotoxins gliotoxin and fumitremorgin. Interestingly, we note that only $A$. fischeri strains

290 synthesized verruculogen, a secondary metabolite that is implicated in human disease and is

291 encoded by the fumitremorgin BGC (Khoufache et al., 2007; Kautsar et al., 2019). Among

292 divergent BGCs that were associated with the absence of a secondary metabolite, we highlight

293 the trypacidin and fumagillin/pseurotin secondary metabolites. We found that nonpathogenic

294 close relatives of $A$. fumigatus produced some but not all mycotoxins, which provides novel

295 insight into the unique cocktail of secondary metabolites biosynthesized by A. fumigatus.

297 Gliotoxin. Gliotoxin is a highly toxic compound and known virulence factor in A. fumigatus

298 (Sugui et al., 2007). Nearly identical BGCs encoding gliotoxin are present in all pathogenic $(A$.

299 fumigatus) and nonpathogenic (A. oerlinghausenensis and A. fischeri) strains examined (Fig. 4).

300 Additionally, we found that all examined strains synthesized bisdethiobis(methylthio)gliotoxin a

301 derivative from dithiogliotoxin, involved in the down-regulation of gliotoxin biosynthesis (Dolan 
et al., 2014), one of the main mechanisms of gliotoxin resistance in A. fumigatus (Kautsar et al., 2019).

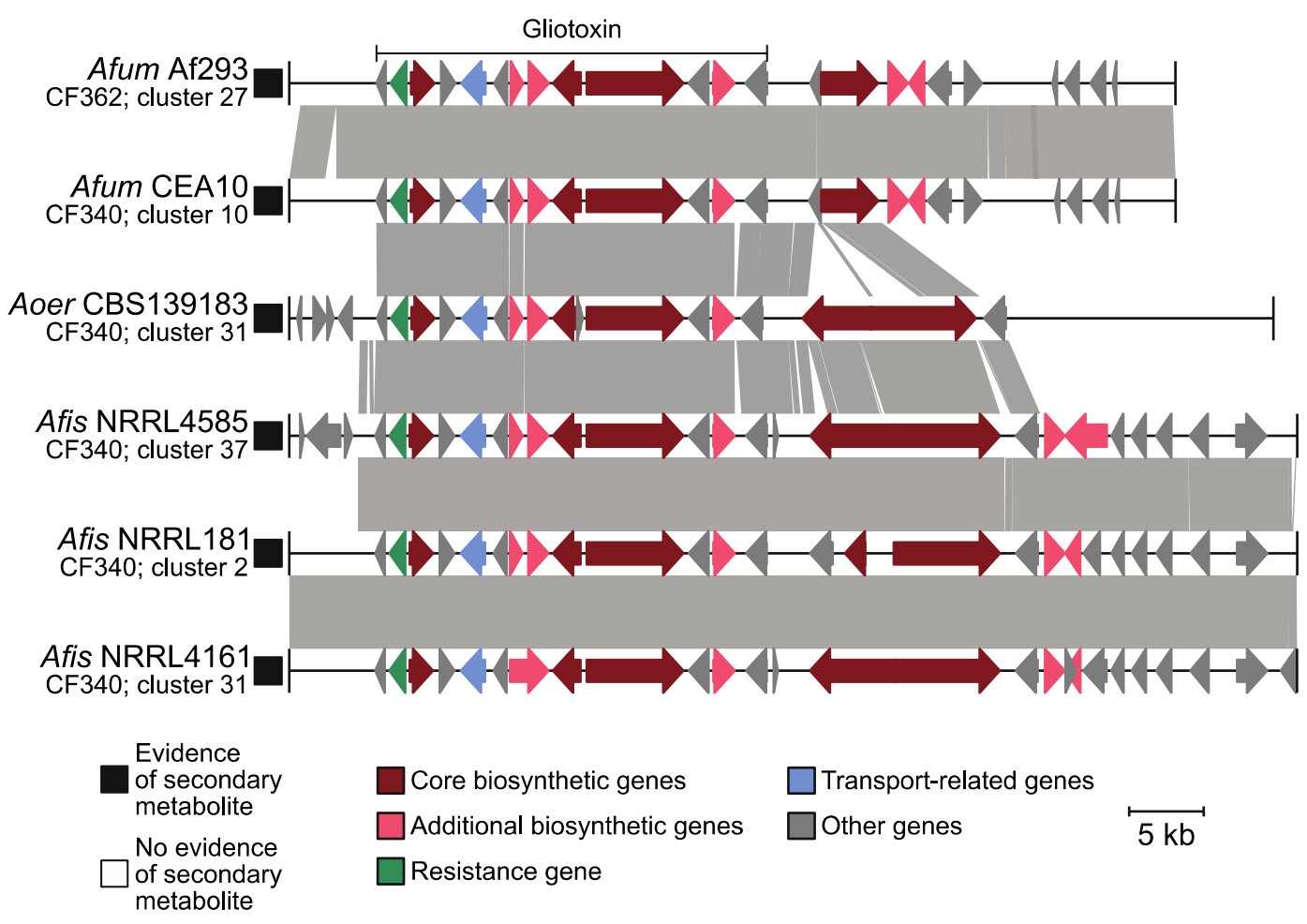

Figure 4. Conservation in the gliotoxin BGC correlates with conserved production of gliotoxin analogs in A. fumigatus and nonpathogenic close relatives. Microsynteny analysis reveals a high degree of conservation in the BGC encoding gliotoxin across all isolates. The known gliotoxin gene cluster boundary is indicated above the A. fumigatus Af293 BGC. Black and white squares correspond to the presence or absence of the associated secondary metabolite, respectively. Genes are drawn as

311 arrows with orientation indicated by the direction of the arrow. Gene function is indicated by gene color.

312 Genus and species names are written using the following abbreviations: Afum: A. fumigatus; Aoer: A.

313 oerlinghausenensis; Afis: A. fischeri. Below each genus and species abbreviation is the cluster family 314 each BGC belongs to and their cluster number. 
317 Fumitremorgin and Verruculogen. Similarly, there is a high degree of conservation in the

318 BGC that encodes fumitremorgin across all strains (Fig. 5). Fumitremorgins have known

319 antifungal activity, are lethal to brine shrimp, and are implicated in inhibiting mammalian

320 proteins responsible for resistance to anticancer drugs in mammalian cells (Raffa and Keller,

321 2019). We found that conservation in the fumitremorgin BGC is associated with the production

322 of fumitremorgins in all isolates examined. The fumitremorgin BGC is also responsible for the

323 production of verruculogen, which is implicated to aid in A. fumigatus pathogenicity by changing

324 the electrophysical properties of human nasal epithelial cells (Khoufache et al., 2007).

325 Interestingly, we found that only A. fischeri strains produced verruculogen under the conditions

326 we analyzed.

328 Trypacidin. Examination of the trypacidin BGC, which encodes a spore-borne and cytotoxic

329 secondary metabolite, revealed a conserved cluster found in four pathogenic and

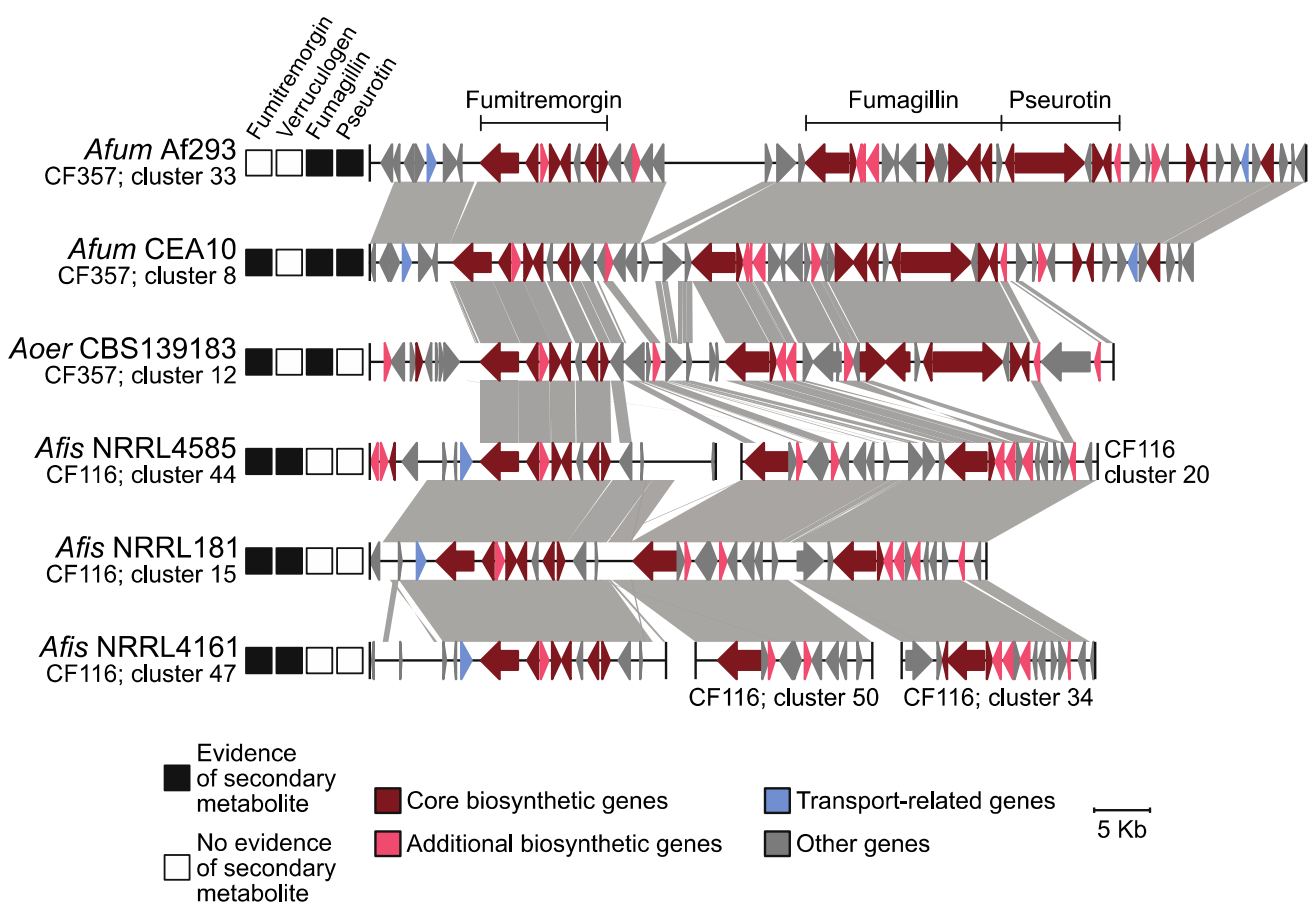


332 Figure 5. Conservation and divergence in the locus encoding the fumitremorgin and intertwined

333 fumagillin/pseurotin BGCs. Microsynteny analysis reveals conservation in the fumitremorgin BGC

334 across all isolates. Interestingly, only A. fischeri strains synthesize verruculogen, a secondary metabolite

335 also biosynthesized by the fumitremorgin BGC. In contrast, the intertwined fumagillin/pseurotin BGCs are

336 conserved between A. fumigatus and A. oerlinghausenensis but divergent in A. fischeri. BGC

337 conservation and divergence is associated with the presence and absence of a secondary metabolite,

338 respectively. The same convention used in Fig. 4 is used to depict evidence of a secondary metabolite,

339 represent genes and broad gene function, genus and species abbreviations, and BGC cluster families

340 and cluster numbers.

$343139183^{\mathrm{T}}$, and A. fischeri NRRL 181 (Fig. S8). Furthermore, we found that three of these four

344 isolates (except $A$. fischeri NRRL 181) biosynthesized a trypacidin analog,

345 monomethylsulochrin. Examination of the microsynteny of the trypacidin BGC revealed that it

346 was conserved across all four genomes with the exception $A$. fischeri NRRL 181, which lacked a

347 RING (Really Interesting New Gene) finger gene. Interestingly, RING finger proteins can

348 mediate gene transcription (Poukka et al., 2000). We confirmed the absence of the RING finger

349 protein by performing a sequence similarity search with the A. fumigatus Af293 RING finger

350 protein (AFUA_4G14620; EAL89333.1) against the A. fischeri NRRL 181 genome. In the

351 homologous locus in A. fischeri, we found no significant blast hit for the first 23 nucleotides of

352 the RING finger gene suggestive of pseudogenization. Taken together, we hypothesize that

353 presence/absence polymorphisms or a small degree of sequence divergence between otherwise

354 homologous BGCs may be responsible for the presence or absence of a toxic secondary

355 metabolite in $A$. fischeri. 
358 revealed that fumagillin has undergone substantial sequence divergence and that pseurotin is

359 absent from strains of $A$. fischeri. The fumagillin/pseurotin BGCs are under the same regulatory

360 control (Wiemann et al., 2013) and biosynthesize secondary metabolites that cause cellular

361 damage during host infection (fumagillin (Guruceaga et al., 2019)) and inhibit immunoglobulin

362 E production (pseurotin (Ishikawa et al., 2009)). Microsynteny of the fumagillin BGC reveals

363 high sequence conservation between A. fumigatus and A. oerlinghausenensis; however, sequence

364 divergence was observed between A. oerlinghausenensis and A. fischeri (Fig. 5). Accordingly,

365 fumagillin production was only observed in $A$. fumigatus and $A$. oerlinghausenensis and not in $A$.

366 fischeri. Similarly, the pseurotin BGC is conserved between $A$. fumigatus and $A$.

367 oerlinghausenensis. Rather than sequence divergence, no sequence similarity was observed in

368 the region of the pseurotin cluster in A. fischeri, which may be due to an indel event.

369 Accordingly, no pseurotin production was observed among $A$. fischeri strains. Despite sequence

370 conservation between A. fumigatus and A. oerlinghausenensis, no evidence of pseurotin

371 biosynthesis was observed in A. oerlinghausenensis, which suggests regulatory decoupling of the

372 intertwined fumagillin/pseurotin BGC. Altogether, these results show a striking correlation

373 between sequence divergence and the production (or absence) of secondary metabolites

374 implicated in human disease among A. fumigatus and nonpathogenic closest relatives.

\section{Discussion}

377 Aspergillus fumigatus is a major fungal pathogen nested within a clade (known as section

378 Fumigati) of at least 60 other species, the vast majority of which are nonpathogenic (Steenwyk et

379 al., 2019; Rokas et al., 2020a). Currently, it is thought that the ability to cause human disease 
evolved multiple times among species in section Fumigati (Rokas et al., 2020a). Secondary metabolites contribute to the success of the major human pathogen A. fumigatus in the host environment (Raffa and Keller, 2019) and are therefore "cards" of virulence (Casadevall, 2007;

383 Knowles et al., 2020). However, whether the closest relatives of A. fumigatus, $A$.

384 oerlinghausenensis and A. fischeri, both of which are nonpathogenic, biosynthesize secondary

385 metabolites implicated in the ability of $A$. fumigatus to cause human disease remained largely

unknown. By examining genomic and chemical variation between and within A. fumigatus and its closest nonpathogenic relatives, we identified both conservation and divergence (including within species heterogeneity) in BGCs and secondary metabolite profiles (Fig. 1-5, S4, S6-9; Table 1, S1, S3). Examples of conserved BGCs and secondary metabolites include the major

390 virulence factor, gliotoxin (Fig. 4), as well as several others (Fig. 5, S8; Table 1, S1, S3);

391 examples of BGC and secondary metabolite heterogeneity or divergence include pseurotin,

392 fumagillin, and several others (Fig. 5; Table 1, S1, S3). Lastly, we found that the fumitremorgin

393 BGC, which biosynthesizes fumitremorgin in all three species, is also associated with

394 verruculogen biosynthesis in A. fischeri strains (Fig. 5).

396 One of the surprising findings of our study was that although $A$. oerlinghausenensis and $A$.

397 fumigatus are evolutionarily more closely related to each other than to A. fischeri (Fig. 1), A.

398 oerlinghausenensis and A. fischeri appear to be more similar to each other than to A. fumigatus in

399 BGC composition, gene family content, and secondary metabolite profiles. The power of

400 pathogen-nonpathogen comparative genomics is best utilized when examining closely related

401 species (Fedorova et al., 2008; Jackson et al., 2011; Moran et al., 2011; Mead et al., 2019a;

402 Rokas et al., 2020a). By sequencing genomes from the closest known nonpathogenic relatives of 
404 additional strains of $A$. fischeri, we provide a powerful resource to study the evolution of $A$.

405 fumigatus pathogenicity.

407 Our finding that $A$. oerlinghausenensis and $A$. fischeri shares more gene families and BGCs with 408 each other than they do with A. fumigatus (Fig. 1C, 2, S4, S5, S9) suggests that the evolutionary 409 trajectory of the A. fumigatus ancestor was marked by gene loss. We hypothesize that there were 410 two rounds of gene family and BGC loss in the A. fumigatus stem lineage: (1) gene families and

411 BGCs were lost in the common ancestor of A. fumigatus and A. oerlinghausenensis and (2)

412 additional losses occurred in the A. fumigatus ancestor. In addition to losses, we note that 548

413 and 16 gene families and BGCs are unique to A. fumigatus, which may have resulted from

414 genetic innovation (e.g., de novo gene formation) or unique gene family and BGC retention (Fig.

4152 , S9). In line with the larger number of shared BGCs between $A$. oerlinghausenensis and $A$.

416 fischeri, we found their secondary metabolite profiles were also more similar (Fig. 3, S7).

417 Notably, the evolutionary rate of the internal branch leading to the A. fumigatus common

418 ancestor is much higher than those in the rest of the branches in our genome-scale phylogeny

419 (Fig. S2B), suggesting that the observed gene loss and gene gain / retention events specific to $A$.

420 fumigatus may be part of a wider set of evolutionary changes in the A. fumigatus genome. More

421 broadly, these results suggest that comparisons of the pathogen A. fumigatus against either the

422 non-pathogen $A$. oerlinghausenensis (this manuscript) or the non-pathogen $A$. fischeri ((Mead et

423 al., 2019a; Knowles et al., 2020) and this manuscript) will both be instructive in understanding

424 the evolution of $A$. fumigatus pathogenicity. 
426 When studying Aspergillus pathogenicity, it is important to consider any genetic and phenotypic

427 heterogeneity between strains of a single species (Kowalski et al., 2016, 2019; Keller, 2017; Ries

428 et al., 2019; Bastos et al., 2020; Santos et al., 2020). Our finding of strain heterogeneity among

429 gene families, BGCs, and secondary metabolites in A. fumigatus and A. fischeri (Fig. 1-3, S4, S5,

430 S7, S9) suggests considerable strain-level diversity in each species. For example, we found

431 secondary metabolite profile strain heterogeneity was greater in A. fumigatus than A. fischeri

432 (Fig. S7B-E). These results suggest that strain specific secondary metabolite profiles may play a

433 role in variation of pathogenicity among A. fumigatus strains. More broadly, our finding supports

434 the hypothesis that strain-level diversity is an important parameter when studying pathogenicity

435 (Kowalski et al., 2016, 2019; Keller, 2017; Ries et al., 2019; Bastos et al., 2020; Santos et al.,

436 2020).

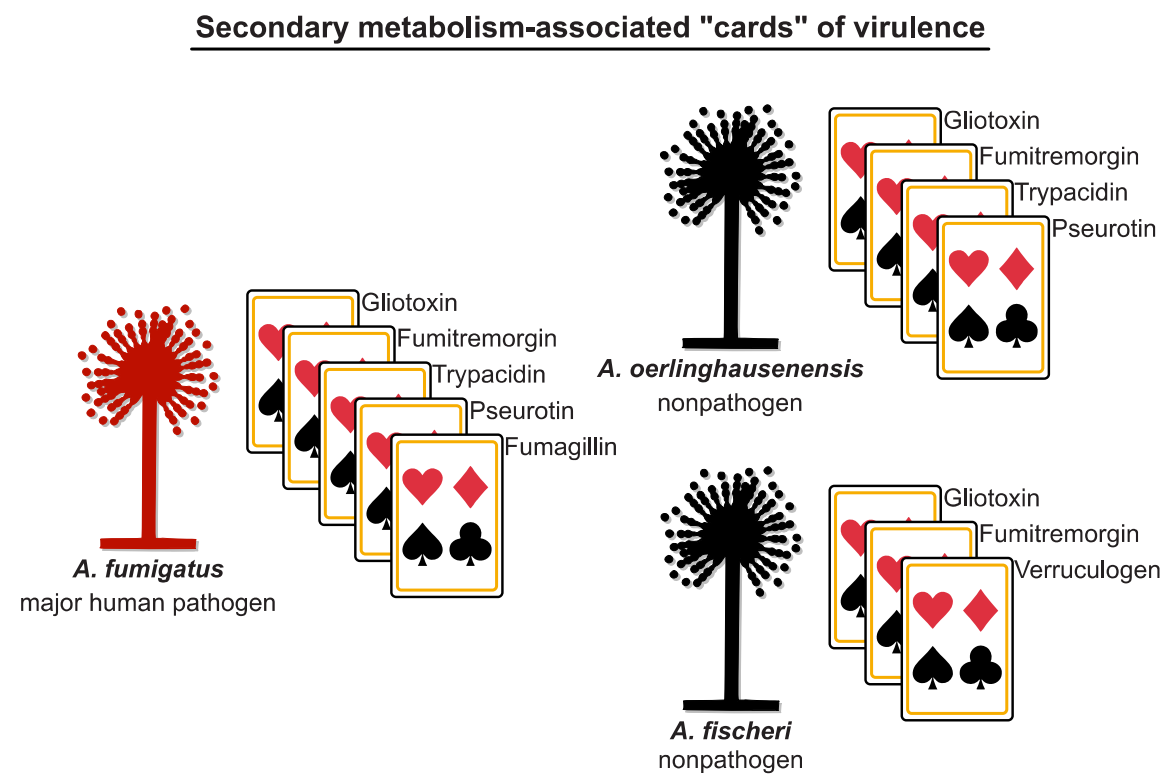

Figure 6. Secondary metabolism-associated "cards" of virulence among A. fumigatus and close

441 relatives. Secondary metabolites contribute to the "hand of cards"' that enable A. fumigatus to cause 
442 disease. Here, we show that the nonpathogenic closest relatives of $A$. fumigatus possess a subset of the

443 A. fumigatus secondary metabolism-associated cards of virulence. We hypothesize that the unique

444 combination of cards of $A$. fumigatus contributes to its pathogenicity and that the cards in $A$.

445 oerlinghausenensis and $A$. fischeri (perhaps in combination with other non-secondary-metabolism-

446 associated cards, such as thermotolerance) are insufficient to cause disease. Pathogenic and

447 nonpathogenic species are shown in red and black, respectively. Cartoons of Aspergillus species were

448 obtained from WikiMedia Commons (source: M. Piepenbring) and modified in accordance with the

449 Creative Commons Attribution-Share Alike 3.0 Unported license

450 (https://creativecommons.org/licenses/by-sa/3.0/deed.en).

452 Secondary metabolites contribute to A. fumigatus virulence through diverse processes including

453 suppressing the human immune system and damaging tissues (Table 1). Interestingly, we found

454 that the nonpathogens A. oerlinghausenensis and A. fischeri produced several secondary

455 metabolites implicated in the ability of A. fumigatus human disease, such gliotoxin, trypacidin,

456 verruculogen, and others (Fig. 4, 5, S8; Table 1, S3). Importantly, our work positively identified

457 secondary metabolites for many structural classes implicated in a previous taxonomic study

458 (Samson et al., 2007). These results suggest that several of the secondary metabolism-associated

459 cards of virulence present in A. fumigatus are conserved in closely related nonpathogens

460 (summarized in Fig. 6). Interestingly, disrupting the ability of A. fumigatus to biosynthesize

461 gliotoxin attenuates but does not abolish virulence (Sugui et al., 2007; Dagenais and Keller,

462 2009; Keller, 2017), whereas disruption of the ability of $A$. fischeri NRRL 181 to biosynthesize

463 secondary metabolites, including gliotoxin, does not appear to influence virulence (Knowles et

464 al., 2020). Our findings, together with previous studies, support the hypothesis that individual

465 secondary metabolites are "cards" of virulence in a larger "hand" that A. fumigatus possesses. 


\section{Methods}

\section{Strain acquisition, DNA extraction, and sequencing}

469 Two strains of Aspergillus fischeri (NRRL 4161 and NRRL 4585) were acquired from the

470 Northern Regional Research Laboratory (NRRL) at the National Center for Agricultural

471 Utilization Research in Peoria, Illinois, while one strain of Aspergillus oerlinghausenensis (CBS

$472139183^{\mathrm{T}}$ ) was acquired from Westerdijk Fungal Biodiversity Institute, Utrecht, The Netherlands.

473 These strains were grown in $50 \mathrm{ml}$ of liquid yeast extract soy peptone dextrose (YESD) medium.

474 After approximately seven days of growth on an orbital shaker (100 rpm) at room temperature,

475 the mycelium was harvested by filtering the liquid media through a Corning ${ }^{\circledR}, 150 \mathrm{ml}$ bottle top,

$476 \quad 0.22 \mu \mathrm{m}$ sterile filter and washed with autoclaved distilled water. All subsequent steps of DNA

477 extraction from the mycelium were performed following protocols outlined previously (Mead et

478 al., 2019b). The genomic DNA from these three strains was sequenced using a NovaSeq S4 at

479 the Vanderbilt Technologies for Advanced Genomes facility (Nashville, Tennessee, US) using

480 paired-end sequencing (150 bp) strategy with the Illumina TruSeq library kit.

\section{Genome assembly, quality assessment, and annotation}

483 To assemble and annotate the three newly sequenced genomes, we first quality-trimmed raw

484 sequence reads using Trimmomatic, v0.36 (Bolger et al., 2014) using parameters described

485 elsewhere (ILLUMINACLIP:TruSeq3-PE.fa:2:30:10, leading:10, trailing:10,

486 slidingwindow:4:20, minlen:50) (Steenwyk and Rokas, 2017). The resulting paired and unpaired

487 quality-trimmed reads were used as input to the SPAdes, v3.11.1 (Bankevich et al., 2012),

488 genome assembly algorithm with the 'careful' parameter and the 'cov-cutoff' set to 'auto'. 
490 We evaluated the quality of our newly assembled genomes, using metrics based on continuity of

491 assembly and gene-content completeness. To evaluate genome assemblies by scaffold size, we

492 calculated the N50 of each assembly (or the shortest contig among the longest contigs that

493 account for 50\% of the genome assembly's length) (Yandell and Ence, 2012). To determine

494 gene-content completeness, we implemented the BUSCO, v2.0.1 (Waterhouse et al., 2018),

495 pipeline using the 'genome' mode. In this mode, the BUSCO pipeline examines assembly

496 contigs for the presence of near-universally single copy orthologous genes (hereafter referred to

497 as BUSCO genes) using a predetermined database of orthologous genes from the OrthoDB, v9

498 (Waterhouse et al., 2013). We used the OrthoDB database for Pezizomycotina (3,156 BUSCO

499 genes). Each BUSCO gene is determined to be present in a single copy, as duplicate sequences,

500 fragmented, or missing. Our analyses indicate the newly sequenced and assembled genomes have

501 high gene-content completeness and assembly continuity (average percent presence of BUSCO

502 genes: $98.80 \pm 0.10 \%$; average N50: 451,294.67 $\pm 9,696.11$; Fig. S1). These metrics suggest

503 these genomes are suitable for comparative genomic analyses.

505 To predict gene boundaries in the three newly sequenced genomes, we used the MAKER,

506 v2.31.10, pipeline (Holt and Yandell, 2011) which, creates consensus predictions from the

507 collective evidence of multiple ab initio gene prediction software. Specifically, we created

508 consensus predictions from SNAP, v2006-07-28 (Korf, 2004), and AUGUSTUS, v3.3.2 (Stanke

509 and Waack, 2003), after training each algorithm individually on each genome. To do so, we first

510 ran MAKER using protein evidence clues from five different publicly available annotations of

511 Aspergillus fungi from section Fumigati. Specifically, we used protein homology clues from $A$.

512 fischeri NRRL 181 (GenBank accession: GCA_000149645.2), A. fumigatus Af293 (GenBank 
513 accession: GCA_000002655.1), Aspergillus lentulus IFM 54703 (GenBank accession:

514 GCA_001445615.1), Aspergillus novofumigatus IBT 16806 (GenBank accession:

515 GCA_002847465.1), and Aspergillus udagawae IFM 46973 (GenBank accession:

516 GCA_001078395.1). The resulting gene predictions were used to train SNAP. MAKER was then

517 rerun using the resulting training results. Using the SNAP trained gene predictions, we trained

518 AUGUSTUS. A final set of gene boundary predictions were obtained by rerunning MAKER

519 with the training results from both SNAP and AUGUSTUS.

521 To supplement our data set of newly sequenced genomes, we obtained publicly available ones.

522 Specifically, we obtained genomes and annotations for A. fumigatus Af293 (GenBank accession:

523 GCA_000002655.1), A. fumigatus CEA10 (strain synonym: CBS 144.89 / FGSC A1163;

524 GenBank accession: GCA_000150145.1), A. fumigatus HMR AF 270 GenBank accession:

525 GCA_002234955.1), A. fumigatus Z5 (GenBank accession: GCA_001029325.1), A. fischeri

526 NRRL 181 (GenBank accession: GCA_000149645.2). We also obtained assemblies of the

527 recently published $A$. fischeri genomes for strains IBT 3003 and IBT 3007 (Zhao et al., 2019)

528 which, lacked annotations. We annotated the genome of each strain individually using MAKER

529 with the SNAP and AUGUSTUS training results from a close relative of both strains, A. fischeri

530 NRRL 4161. Altogether, our final data set contained a total of ten genome from three species:

531 four A. fumigatus strains, one $A$. oerlinghausenensis strain, and five $A$. fischeri strains.

\section{Maximum likelihood phylogenetics and Bayesian estimation of divergence times}

534 To reconstruct the evolutionary history among the ten Aspergillus genomes, we implemented a

535 recently developed pipeline (Steenwyk et al., 2019) which, relies on the concatenation-approach 
to phylogenomics (Rokas et al., 2003) and has been successfully used in reconstructing species-

537 level relationships among Aspergillus and Penicillium fungi (Bodinaku et al., 2019; Steenwyk et

538 al., 2019). The first step in the pipeline is to identify single copy orthologous genes in the

539 genomes of interest which, are ultimately concatenated into a larger phylogenomic data matrix.

540 To identify single copy BUSCO genes across all ten Aspergillus genomes, we used the BUSCO

541 pipeline with the Pezizomycotina database as described above. We identified 3,041 BUSCO

542 genes present at a single copy in all ten Aspergillus genomes and created multi-FASTA files for

543 each BUSCO gene that contained the protein sequences for all ten taxa. The protein sequences of

544 each BUSCO gene were individually aligned using Mafft, v7.4.02 (Katoh and Standley, 2013),

545 with the same parameters as described elsewhere (Steenwyk et al., 2019). Nucleotide sequences

546 were then forced onto the protein sequence alignments using a custom Python, v3.5.2

547 (https://www.python.org/), script with BioPython, v1.7 (Cock et al., 2009). The resulting codon-

548 based alignments were trimmed using trimAl, v1.2.rev59 (Capella-Gutierrez et al., 2009), with

549 the 'gappyout' parameter. The resulting trimmed nucleotide alignments were concatenated into a

550 single matrix of 5,602,272 sites and was used as input into IQ-TREE, v1.6.11 (Nguyen et al.,

551 2015). The best-fitting model of substitutions for the entire matrix was determined using

552 Bayesian information criterion values (Kalyaanamoorthy et al., 2017). The best-fitting model

553 was a general time-reversible model with empirical base frequencies that allowed for a

554 proportion of invariable sites and a discrete Gamma model with four rate categories

555 (GTR+I+F+G4) (Tavaré, 1986; Yang, 1994, 1996; Vinet and Zhedanov, 2011). To evaluate

556 bipartition support, we used 5,000 ultrafast bootstrap approximations (Hoang et al., 2018). 
To estimate divergence times among the ten Aspergillus genomes, we used the concatenated data

559 matrix and the resulting maximum likelihood phylogeny from the previous steps as input to

560 Bayesian approach implemented in MCMCTree from the PAML package, v4.9d (Yang, 2007).

561 First, we estimated the substitution rate across the data matrix using a "GTR $+\mathrm{G}$ " model of

562 substitutions (model $=7$ ), a strict clock model, and the maximum likelihood phylogeny rooted on

563 the clade of $A$. fischeri strains. We imposed a root age of 3.69 million years ago according to

564 results from recent divergence time estimates of the split between A. fischeri and A. fumigatus

565 (Steenwyk et al., 2019). We estimated the substitution rate to be 0.005 substitutions per one

566 million years. Next, the likelihood of the alignment was approximated using a gradient and

567 Hessian matrix. To do so, we used previously established time constraints for the split between

568 A. fischeri and A. fumigatus (1.85 to 6.74 million years ago) (Steenwyk et al., 2019). Lastly, we

569 used the resulting gradient and Hessian matrix, the rooted maximum likelihood phylogeny, and

570 the concatenated data matrix to estimate divergence times using a relaxed molecular clock

571 (model $=2)$. We specified the substitution rate prior based on the estimated substitution rate

572 (rgene_gamma $=1$ 186.63). The 'sigma2_gamma' and 'finetune' parameters were set to ' 14.5 '

573 and ' 1 ', respectively. To collect a high-quality posterior probability distribution, we ran a total of

5745.1 million iterations during MCMC analysis which, is 510 times greater than the minimum

575 recommendations (Raftery and Lewis, 1995). Our sampling strategy across the 5.1 million

576 iterations was to discard the first 100,000 results followed by collecting a sample every $500^{\text {th }}$

577 iteration until a total of 10,000 samples were collected. 
580 To identify gene families across the ten Aspergillus genomes, we used a Markov clustering

581 approach. Specifically, we used OrthoFinder, v2.3.8 (Emms and Kelly, 2019). OrthoFinder first

582 conducts a blast all-vs-all using the protein sequences of all ten Aspergillus genomes and NCBI's

583 Blast+, v2.3.0 (Camacho et al., 2009), software. After normalizing blast bit scores, genes are

584 clustered into discrete orthogroups using a Markov clustering approach (van Dongen, 2000). We

585 clustered genes using an inflation parameter of 1.5. The resulting orthogroups were used proxies

586 for gene families.

588 To identify putative biosynthetic gene clusters (BGCs), we used the gene boundaries predictions

589 from the MAKER software as input into antiSMASH, v4.1.0 (Weber et al., 2015). To identify

590 homologous BGCs across the ten Aspergillus genomes, we used the software BiG-SCAPE,

591 v20181005 (Navarro-Muñoz et al., 2020). Based on the Jaccard Index of domain types, sequence

592 similarity among domains, and domain adjacency, BiG-SCAPE calculates a similarity metric

593 between pairwise combinations of clusters where smaller values indicate greater BGC similarity.

594 BiG-SCAPE's similarity metric can then be used as an edge-length in network analyses of

595 cluster similarity. We evaluated networks using an edge-length cutoff from 0.1-0.9 with a step of

$596 \quad 0.1$ (Fig. S4). We found networks with an edge-length cutoff of 0.4-0.6 to be similar and based

597 further analyses on a cutoff of 0.5. For BGCs of interest, we supplemented BiG-SCAPE's

598 approach to identifying homologous BGCs with visualize inspection of microsyteny and blast-

599 based analyses using NCBI's BLAST+, v2.3.0 (Camacho et al., 2009). Similar sequences in

600 microsynteny analyses were defined as at least $100 \mathrm{bp}$ in length, at least 30 percent similarity,

601 and an expectation value threshold of 0.01 . 


\section{Identification and characterization of secondary metabolite production}

\section{General experimental procedures}

605 The ${ }^{1} \mathrm{H}$ NMR data were collected using a JOEL ECS-400 spectrometer, which was equipped

606 with a JOEL normal geometry broadband Royal probe, and a 24-slot autosampler, and operated

607 at $400 \mathrm{MHz}$. HRESIMS experiments utilized either a Thermo LTQ Orbitrap XL mass

608 spectrometer or a Thermo Q Exactive Plus (Thermo Fisher Scientific); both were equipped with

609 an electrospray ionization source. A Waters Acquity UPLC (Waters Corp.) was utilized for both

610 mass spectrometers, using a $\mathrm{BEH} \mathrm{C}_{18}$ column $(1.7 \mu \mathrm{m} ; 50 \mathrm{~mm} \times 2.1 \mathrm{~mm})$ set to a temperature of

$61140^{\circ} \mathrm{C}$ and a flow rate of $0.3 \mathrm{ml} / \mathrm{min}$. The mobile phase consisted of a linear gradient of $\mathrm{CH}_{3} \mathrm{CN}-$

$612 \mathrm{H}_{2} \mathrm{O}$ (both acidified with $0.1 \%$ formic acid), starting at $15 \% \mathrm{CH}_{3} \mathrm{CN}$ and increasing linearly to

$613100 \% \mathrm{CH}_{3} \mathrm{CN}$ over $8 \mathrm{~min}$, with a $1.5 \mathrm{~min}$ hold before returning to the starting condition. The

614 HPLC separations were performed with Atlantis T3 $\mathrm{C}_{18}$ semi-preparative $(5 \mu \mathrm{m} ; 10 \times 250 \mathrm{~mm})$

615 and preparative $(5 \mu \mathrm{m} ; 19 \times 250 \mathrm{~mm})$ columns, at a flow rate of $4.6 \mathrm{ml} / \mathrm{min}$ and $16.9 \mathrm{ml} / \mathrm{min}$,

616 respectively, with a Varian Prostar HPLC system equipped with a Prostar 210 pumps and a

617 Prostar 335 photodiode array detector (PDA), with the collection and analysis of data using

618 Galaxie Chromatography Workstation software. Flash chromatography was performed on a

619 Teledyne ISCO Combiflash Rf 200 and monitored by both ELSD and PDA detectors.

\section{Chemical characterization}

622 To identify the secondary metabolites that were biosynthesized by $A$. fumigatus, $A$.

623 oerlinghausenensis, and $A$. fischeri, these strains were grown as large-scale fermentations to

624 isolate and characterize the secondary metabolites. To inoculate oatmeal cereal media (Old

625 fashioned breakfast Quaker oats), agar plugs from fungal stains grown on potato dextrose agar; 
626 difco (PDA) were excised from the edge of the Petri dish culture and transferred to separate

627 liquid seed media that contained $10 \mathrm{ml}$ YESD broth (2\% soy peptone, $2 \%$ dextrose, and $1 \%$ yeast

628 extract; $5 \mathrm{~g}$ of yeast extract, $10 \mathrm{~g}$ of soy peptone, and $10 \mathrm{~g}$ of D-glucose in $500 \mathrm{ml}$ of deionized

$629 \mathrm{H}_{2} \mathrm{O}$ ) and allowed to grow at $23^{\circ} \mathrm{C}$ with agitation at $100 \mathrm{rpm}$ for three days. The YESD seed

630 cultures of the fungi were subsequently used to inoculate solid-state oatmeal fermentation

631 cultures, which were either grown at room temperature (approximately $23^{\circ} \mathrm{C}$ under $12 \mathrm{~h}$

632 light/dark cycles for 14 days), $30^{\circ} \mathrm{C}$, or $37^{\circ} \mathrm{C}$; all growths at the latter two temperatures were

633 carried out in an incubator (VWR International) in the dark over four days. The oatmeal cultures

634 were prepared in $250 \mathrm{ml}$ Erlenmeyer flasks that contained $10 \mathrm{~g}$ of autoclaved oatmeal (10 $\mathrm{g}$ of

635 oatmeal with $17 \mathrm{ml}$ of deionized $\mathrm{H}_{2} \mathrm{O}$ and sterilized for $15-20$ minutes at $121^{\circ} \mathrm{C}$ ). For all fungal

636 strains three flasks of oatmeal cultures were grown at all three temperatures, except for $A$.

637 oerlinghausenensis (CBS $139183^{\mathrm{T}}$ ) at room temperature and A. fumigatus (Af293) at $37^{\circ} \mathrm{C}$. For

638 CBS $139183^{\mathrm{T}}$, the fungal cultures were grown in four flasks, while for Af293 eight flasks were

639 grown in total. The growths of these two strains were performed differently from the rest because

640 larger amounts of extract were required in order to perform detailed chemical characterization.

641 The cultures were extracted by adding $60 \mathrm{ml}$ of (1:1) $\mathrm{MeOH}-\mathrm{CHCl}_{3}$ to each $250 \mathrm{ml}$ flask,

642 chopping thoroughly with a spatula, and shaking overnight $(\sim 16 \mathrm{~h})$ at $\sim 100 \mathrm{rpm}$ at room

643 temperature. The culture was filtered in vacuo, and $90 \mathrm{ml} \mathrm{CHCl}_{3}$ and $150 \mathrm{ml} \mathrm{H}_{2} \mathrm{O}$ were added to

644 the filtrate. The mixture was stirred for $30 \mathrm{~min}$ and then transferred to a separatory funnel. The

645 organic layer $\left(\mathrm{CHCl}_{3}\right)$ was drawn off and evaporated to dryness in vacuo. The dried organic layer

646 was reconstituted in $100 \mathrm{ml}$ of $(1: 1) \mathrm{MeOH}-\mathrm{CH}_{3} \mathrm{CN}$ and $100 \mathrm{ml}$ of hexanes, transferred to a

647 separatory funnel, and shaken vigorously. The defatted organic layer $\left(\mathrm{MeOH}-\mathrm{CH}_{3} \mathrm{CN}\right)$ was

648 evaporated to dryness in vacuo. 
650 To isolate compounds, the defatted extract was dissolved in $\mathrm{CHCl}_{3}$, absorbed onto Celite 545

651 (Acros Organics), and fractioned by normal phase flash chromatography using a gradient of

652 hexane- $\mathrm{CHCl}_{3}-\mathrm{MeOH}$. Aspergillus fischeri strain NRRL 181 was chemically characterized

653 previously (Knowles et al., 2019; Mead et al., 2019a). A. fumigatus strain Af293, grown at $37^{\circ} \mathrm{C}$,

654 was subjected to a $12 \mathrm{~g}$ column at a flow rate of $30 \mathrm{ml} / \mathrm{min}$ and 61.0 column volumes, which

655 yielded four fractions. Fraction 2 was further purified via preparative HPLC using a gradient

656 system of 30:70 to $100: 0$ of $\mathrm{CH}_{3} \mathrm{CN}-\mathrm{H}_{2} \mathrm{O}$ with $0.1 \%$ formic acid over $40 \mathrm{~min}$ at a flow rate of

$657 \quad 16.9 \mathrm{ml} / \mathrm{min}$ to yield six subfractions. Subfractions 1, 2 and 5, yielded cyclo(L-Pro-L-Leu) (1)

658 (Li et al., 2008) (0.89 mg), cyclo(L-Pro-L-Phe) (2) (Campbell et al., 2009) (0.71 mg), and

659 monomethylsulochrin (3) (Ma et al., 2004) (2.04 mg), which eluted at approximately 5.7, 6.3,

660 and $10.7 \mathrm{~min}$, respectively. Fraction 3 was further purified via preparative HPLC using a

661 gradient system of 40:60 to $65: 35$ of $\mathrm{CH}_{3} \mathrm{CN}-\mathrm{H}_{2} \mathrm{O}$ with $0.1 \%$ formic acid over 30 min at a flow

662 rate of $16.9 \mathrm{ml} / \mathrm{min}$ to yield four subfractions. Subfractions 1 and 2 yielded pseurotin A (4)

663 (Wang et al., 2011) (12.50 mg) and bisdethiobis(methylthio)gliotoxin (5) (Afiyatullov et al.,

$6642005)(13.99 \mathrm{mg})$, which eluted at approximately 7.5 and $8.0 \mathrm{~min}$, respectively.

665

666 A. fumigatus strain CEA10, grown at $37^{\circ} \mathrm{C}$, was subjected to a $4 \mathrm{~g}$ column at a flow rate of 18

$667 \mathrm{ml} / \mathrm{min}$ and 90.0 column volumes, which yielded five fractions. Fraction 1 was purified via

668 preparative HPLC using a gradient system of 50:50 to 100:0 of $\mathrm{CH}_{3} \mathrm{CN}-\mathrm{H}_{2} \mathrm{O}$ with 0.1\% formic

669 acid over $45 \mathrm{~min}$ at a flow rate of $16.9 \mathrm{ml} / \mathrm{min}$ to yield eight subfractions. Subfraction 1, yielded

670 fumagillin (6) (Halász et al., 2000) (1.69 mg), which eluted at approximately 18.5 min. Fraction

6712 was purified via semi-preparative HPLC using a gradient system of 35:65 to 80:20 of $\mathrm{CH}_{3} \mathrm{CN}-$ 
$672 \mathrm{H}_{2} \mathrm{O}$ with $0.1 \%$ formic acid over $30 \mathrm{~min}$ at a flow rate of $4.6 \mathrm{ml} / \mathrm{min}$ to yield 10 subfractions.

673 Subfraction 5 yielded fumitremorgin C (7) (Kato et al., 2009) (0.25 mg), which eluted at

674 approximately $15.5 \mathrm{~min}$. Fraction 3 was purified via preparative HPLC using a gradient system

675 of 40:60 to 100:0 of $\mathrm{CH}_{3} \mathrm{CN}-\mathrm{H}_{2} \mathrm{O}$ with $0.1 \%$ formic acid over $30 \mathrm{~min}$ at a flow rate of 16.9

$676 \mathrm{ml} / \mathrm{min}$ to yield nine subfractions. Subfraction 2 yielded pseurotin A (4) (1.64 mg), which eluted

677 at approximately $7.3 \mathrm{~min}$.

678

679 Aspergillus oerlinghausenensis strain CBS $139183^{\mathrm{T}}$, grown at RT, was subjected to a $4 \mathrm{~g}$ column

680 at a flow rate of $18 \mathrm{ml} / \mathrm{min}$ and 90 column volumes, which yielded 4 fractions. Fraction 3 was

681 further purified via preparative $\mathrm{HPLC}$ using a gradient system of $35: 65$ to $70: 30$ of $\mathrm{CH}_{3} \mathrm{CN}-\mathrm{H}_{2} \mathrm{O}$

682 with $0.1 \%$ formic acid over $40 \mathrm{~min}$ at a flow rate of $16.9 \mathrm{ml} / \mathrm{min}$ to yield 11 subfractions.

683 Subfractions 3 and 10 yielded spiro [5H,10H-dipyrrolo[1,2-a:1',2'-d]pyrazine-2-(3H),2'-

684 [2H]indole]-3',5,10(1'H)-trione (8) (Wang et al., 2008) (0.64 mg) and helvolic acid (9) (Zhao et

685 al., 2010) (1.03 mg), which eluted at approximately 11.5 and $39.3 \mathrm{~min}$, respectively. (see NMR

686 supporting information; figshare: 10.6084/m9.figshare.12055503).

\section{Metabolite profiling by mass spectrometry}

689 The metabolite profiling by mass spectrometry, also known as dereplication, was performed as

690 stated previously (El-Elimat et al., 2013). Briefly, ultraperformance liquid chromatography-

691 photodiode array-electrospray ionization high resolution tandem mass spectrometry (UPLC-

692 PDA-HRMS-MS/MS) was utilized to monitor for secondary metabolites across all strains

693 (Af293, CEA10, CEA17, CBS 139183 ${ }^{\mathrm{T}}$, NRRL 181, NRRL 4161, and NRRL 4585). Utilizing

694 positive-ionization mode, ACD MS Manager with add-in software IntelliXtract (Advanced 
695 Chemistry Development, Inc.; Toronto, Canada) was used for the primary analysis of the UPLC-

696 MS chromatograms. The data from 19 secondary metabolites are provided in the Supporting

697 Information (see Dereplication table; figshare: 10.6084/m9.figshare.12055503), which for each

698 secondary metabolite lists: molecular formula, retention time, UV-absorption maxima, high-

699 resolution full-scan mass spectra, and MS-MS data (top 10 most intense peaks).

701 Metabolomics analyses

702 Principal component analysis (PCA) analysis was performed on the UPLC-MS data. Untargeted

703 UPLC-MS datasets for each sample were individually aligned, filtered, and analyzed using

704 MZmine 2.20 software (https://sourceforge.net/projects/mzmine/) (Pluskal et al., 2010). Peak

705 detection was achieved using the following parameters, A. fumigatus at (Af293, CEA10, and

706 CEA17): noise level (absolute value), $1 \times 10^{6}$; minimum peak duration, $0.05 \mathrm{~min}$; $\mathrm{m} / \mathrm{z}$ variation

707 tolerance, 0.05 ; and $\mathrm{m} / \mathrm{z}$ intensity variation, 20\%; A fischeri (NRRL 181, NRRL 4161, and

708 NRRL 4585): noise level (absolute value), $1 \times 10^{6}$; minimum peak duration, $0.05 \mathrm{~min} ; \mathrm{m} / \mathrm{z}$

709 variation tolerance, 0.05 ; and $\mathrm{m} / \mathrm{z}$ intensity variation, 20\%; and all strains (Af293, CEA10,

710 CEA17, CBS 139183 ${ }^{\mathrm{T}}$, NRRL 181, NRRL 4161, and NRRL 4585): noise level (absolute value),

$7117 \times 10^{5} ;$ minimum peak duration, $0.05 \mathrm{~min} ; \mathrm{m} / \mathrm{z}$ variation tolerance, 0.05 ; and $\mathrm{m} / \mathrm{z}$ intensity

712 variation, 20\%. Peak list filtering and retention time alignment algorithms were used to refine

713 peak detection. The join algorithm integrated all sample profiles into a data matrix using the

714 following parameters: $\mathrm{m} / \mathrm{z}$ and retention time balance set at 10.0 each, $\mathrm{m} / \mathrm{z}$ tolerance set at 0.001 ,

715 and RT tolerance set at 0.5 mins. The resulting data matrix was exported to Excel (Microsoft) for

716 analysis as a set of $\mathrm{m} / \mathrm{z}$ - retention time pairs with individual peak areas detected in triplicate

717 analyses. Samples that did not possess detectable quantities of a given marker ion were assigned 
718 a peak area of zero to maintain the same number of variables for all sample sets. Ions that did not

719 elute between 2 and 8 minutes and/or had an $\mathrm{m} / \mathrm{z}$ ratio less than 200 or greater than 800 Da were

720 removed from analysis. Relative standard deviation was used to understand the quantity of

721 variance between the technical replicate injections, which may differ slightly based on

722 instrument variance. A cutoff of 1.0 was used at any given $\mathrm{m} / \mathrm{z}$ - retention time pair across the

723 technical replicate injections of one biological replicate, and if the variance was greater than the

724 cutoff, it was assigned a peak area of zero. Final chemometric analysis, data filtering (Caesar et

725 al., 2018) and PCA was conducted using Sirius, v10.0 (Pattern Recognition Systems AS)

726 (Kvalheim et al., 2011), and dendrograms were created with Python. The PCA scores plots were

727 generated using data from either the three individual biological replicates or the averaged

728 biological replicates of the fermentations. Each biological replicate was plotted using averaged

729 peak areas obtained across four replicate injections (technical replicates).

$731 \quad$ Data Availability

732 Sequence reads and associated genome assemblies generated in this project are available in

733 NCBI's GenBank database under the BioProject PRJNA577646. Additional descriptions of the

734 genomes including predicted gene boundaries will become available through the Figshare

735 repository 10.6084/m9.figshare.12055503 upon publication. The Figshare repository is also

736 populated with other data generated from genomic and natural products analysis. Among

737 genomic analyses, we provide information about predicted BGCs, results associated with

738 network-based clustering of BGCs into cluster families, phylogenomic data matrices, and others.

739 Among natural products analysis, we provide information that supports methods, and results,

740 including NMR spectra. 


\section{$742 \quad$ Funding}

743 JLS and AR are supported by the Howard Hughes Medical Institute through the James H.

744 Gilliam Fellowships for Advanced Study program. AR has additional support from a Discovery

745 Grant from Vanderbilt University. GHG is supported by the Brazilian funding agencies

746 Fundacão de Amparo a Pesquisa do Estado de São Paulo (FAPESP 2016/07870-9) and Conselho

747 Nacional de Desenvolvimento Cientıfico e Tecnologico (CNPq). NHO is supported by the

748 National Cancer Institute (P01 CA125066). SLK and CDR were supported in part by the

749 National Institutes of Health via the National Center for Complementary and Integrative Health

750 (F31 AT010558) and the National Institute of General Medical Sciences (T34 GM113860),

751 respectively.

752

\section{Acknowledgements}

754 We thank the labs of Rokas, Oberlies, and Goldman for helpful discussion and support of this

755 work. 
Table 1. Select $A$. fumigatus secondary metabolites implicated in human disease

\begin{tabular}{|c|c|c|c|c|c|c|c|c|c|}
\hline & \multirow{3}{*}{ Function } & \multirow{3}{*}{ Reference(s) } & \multicolumn{7}{|c|}{ Evidence of secondary metabolite } \\
\hline & & & \multicolumn{3}{|c|}{ A. fumigatus } & \multirow{2}{*}{$\begin{array}{c}\text { A. } \\
\text { oerlinghause } \\
\text { nensis } \\
\text { CBS } 139183^{\mathrm{T}}\end{array}$} & \multicolumn{3}{|c|}{ A. fischeri } \\
\hline & & & Af293 & CEA10 & CEA17 & & NRRL 181 & NRRL 4585 & NRRL 4161 \\
\hline Gliotoxin & $\begin{array}{l}\text { Inhibits host } \\
\text { immune } \\
\text { response }\end{array}$ & $\begin{array}{c}\text { (Sugui et al., } \\
\text { 2007) }\end{array}$ & + & + & + & 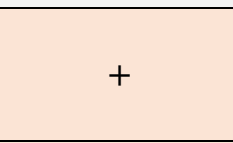 & 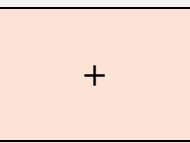 & 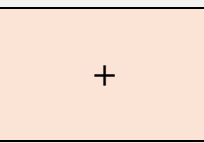 & 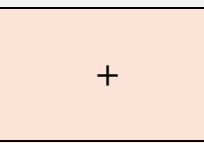 \\
\hline Fumitremorgin & $\begin{array}{l}\text { Inhibits the } \\
\text { breast cancer } \\
\text { resistance } \\
\text { protein }\end{array}$ & $\begin{array}{c}\text { (González- } \\
\text { Lobato et al., } \\
\text { 2010) }\end{array}$ & - & + & - & + & + & + & + \\
\hline Verruculogen & $\begin{array}{c}\text { Changes } \\
\text { electrophysical } \\
\text { properties of } \\
\text { human nasal } \\
\text { epithelial cells } \\
\end{array}$ & $\begin{array}{c}\text { (Khoufache et } \\
\text { al., 2007) }\end{array}$ & - & - & - & + & + & + & + \\
\hline Trypacidin & $\begin{array}{c}\text { Damages lung } \\
\text { cell tissues }\end{array}$ & $\begin{array}{c}\text { (Gauthier et } \\
\text { al., 2012) }\end{array}$ & + & + & - & + & - & - & - \\
\hline Pseurotin & $\begin{array}{c}\text { Inhibits } \\
\text { immunoglobulin } \\
\text { E }\end{array}$ & $\begin{array}{l}\text { (Ishikawa et } \\
\text { al., 2009) }\end{array}$ & + & + & + & + & - & - & - \\
\hline Fumagillin & $\begin{array}{l}\text { Inhibits } \\
\text { neutrophil } \\
\text { function }\end{array}$ & $\begin{array}{c}\text { (Fallon et al., } \\
2010,2011 \text { ) }\end{array}$ & + & + & + & - & - & - & - \\
\hline
\end{tabular}

$757 \quad$ A list of select secondary metabolites implicated in human disease and their functional role are described here. All secondary

758 metabolites listed or analogs thereof were identified during secondary metabolite profiling. '+' (orange) and '-' (blue) indicate

759 if the secondary metabolite was or was not produced by a strain of $\boldsymbol{A}$. fumigatus or its two closest relatives. 


\section{Supplementary legends}

761 Fig. S1. Metrics of genomes assembly quality and number of predicted gene. Genome size,

762 N50, number of genes, number of scaffolds and percent single copy BUSCO genes (scBUSCO)

763 are depicted here. Examination of metrics reveal genomes are of sufficient quality for

764 comparative genomics purposes. Of concern, we noted A. oerlinghausenensis CBS $139183^{\mathrm{T}}$ was

765 assembled into 5,300 contigs; however, we evaluated the assembly's N50 value and gene content

766 completeness (461,327 base pairs and 98.90\% BUSCO genes present in single copy,

767 respectively) and found the A. oerlinghausenensis genome is suitable for comparative genomic

768 analyses.

769

770 Fig. S2. A reconstructed evolutionary history and timetree of $\boldsymbol{A}$. fumigatus and its closest

771 relatives. (top) Divergence times were estimated using a concatenated matrix of 3,041 genes

$772(5,602,272$ sites $)$. Blue bars at each node correspond to the $95 \%$ divergence time confidence

773 interval. Divergence times and confidence intervals for each internode are shown on the right

774 side of the figure. (bottom) A phylogeny where branch lengths represent substitutions per site

775 rather than geologic time. A cladogram is drawn to the right of the phylogeny to clarify

776 divergences where branch lengths are short (e.g., among strains of A. fischeri).

778 Fig. S3. Moderate edge cut-off lengths resulted in qualitatively similar networks. Networks

779 using edge cut-offs ranging from $0.1-0.9$ with a step of 0.1 were evaluated. Networks from 0.4 to

$780 \quad 0.6$ were qualitatively similar. Thus, we used cluster families inferred from the network using an

781 edge cut-off of 0.5 . 
Fig. S4. Species and strain heterogeneity among BGC cluster presence and absence. (A)

784 Examination of the number of strains in each cluster family reveal a wide variation. For example,

785 all 10 genomes are represented in 17 cluster families; in contrast, 18 cluster families have only

786 one BGC. (B) Species occupancy among cluster families reveal A. fischeri has the largest

787 number of unique BGCs followed by A. fumigatus and A. oerlinghausenensis. (C) Strain-level

788 presence and absence patterns among cluster families reveal substantial species and strain

789 heterogeneity. Cluster family identifiers are shown along the x-axis. Genus and species names

790 are written using the following abbreviations: Afum: A. fumigatus; Aoer: A. oerlinghausenensis;

791 Afis: A. fischeri.

Fig. S5. Gene family presence and absence follows a similar pattern to BGCs. Orthogroups

794 were used as proxies for gene families. A strain-level UpSet plot for gene family presence and 795 absence patterns reveals heterogeneity among strains.

Fig. S6. Structures of isolated fungal metabolites. Secondary metabolites produced in

798 sufficient quantity were isolated for structural determination. The structures of compounds are

799 correlated to each strain from where they were isolated.

Fig. S7. Principal component analysis of BGC presence and absence and secondary metabolite profiles mirror one another. (A) Principal component analysis of BGC presence and absence reveal that each species is distinct from the other. Furthermore, $A$.

804 oerlinghausenensis is between A. fischeri and A. fumigatus. (B, C) Broadly, similar patterns of 
805 species relationships in principal component space are observed among all metabolites produced

806 at $37^{\circ} \mathrm{C}$ and $30^{\circ} \mathrm{C}$. (D, E) Similar results were observed for isolatable metabolites.

808 Fig. S8. Small sequence divergences in the trypacidin BGC are associated with the

809 production of trypacidin or the lack thereof. The trypacidin BGC is found in $A$. fumigatus

810 strains Af293 and CEA10, A. oerlinghausenensis CBS 139183 ${ }^{\mathrm{T}}$, and A. fischeri NRRL 181.

811 Evidence of trypacidin biosynthesis is found in all isolates with the exception of $A$. fischeri

812 NRRL 181. The absence of trypacidin biosynthesis is associated with the absence of a RING

813 finger gene in $A$. fischeri NRRL 181. Black and white squares correspond to the presence or

814 absence of the associated secondary metabolite, respectively. Genus and species names are

815 written using the following abbreviations: Afum: A. fumigatus; Aoer: A. oerlinghausenensis; Afis:

816 A. fischeri. 


\section{$\underline{\text { References }}$}

818 Abad, A., Victoria Fernández-Molina, J., Bikandi, J., Ramírez, A., Margareto, J., Sendino, J., et

Afiyatullov, S. S., Kalinovskii, A. I., Pivkin, M. V., Dmitrenok, P. S., and Kuznetsova, T. A.

$$
\text { Compd. 41, 236-238. doi:10.1007/s10600-005-0122-y. }
$$

825 Bankevich, A., Nurk, S., Antipov, D., Gurevich, A. A., Dvorkin, M., Kulikov, A. S., et al. Sequencing. J. Comput. Biol. 19, 455-477. doi:10.1089/cmb.2012.0021.

Bastos, R. W., Valero, C., Silva, L. P., Schoen, T., Drott, M., Brauer, V., et al. (2020).

Benedict, K., Jackson, B. R., Chiller, T., and Beer, K. D. (2019). Estimation of Direct Healthcare

834 Bodinaku, I., Shaffer, J., Connors, A. B., Steenwyk, J. L., Biango-Daniels, M. N., Kastman, E. K., et al. (2019). Rapid Phenotypic and Metabolomic Domestication of Wild Penicillium Molds on Cheese. MBio 10. doi:10.1128/mBio.02445-19. sequence data. Bioinformatics 30, 2114-2120. doi:10.1093/bioinformatics/btu170.

839 Bongomin, F., Gago, S., Oladele, R., and Denning, D. (2017). Global and Multi-National 
Prevalence of Fungal Diseases_Estimate Precision. J. Fungi 3, 57. doi:10.3390/jof3040057.

842 Caesar, L. K., Kvalheim, O. M., and Cech, N. B. (2018). Hierarchical cluster analysis of

843 technical replicates to identify interferents in untargeted mass spectrometry metabolomics. Anal. Chim. Acta 1021, 69-77. doi:10.1016/j.aca.2018.03.013.

845 Camacho, C., Coulouris, G., Avagyan, V., Ma, N., Papadopoulos, J., Bealer, K., et al. (2009). BLAST+: architecture and applications. BMC Bioinformatics 10, 421. doi:10.1186/1471-

Campbell, J., Lin, Q., Geske, G. D., and Blackwell, H. E. (2009). New and Unexpected Insights into the Modulation of LuxR-Type Quorum Sensing by Cyclic Dipeptides. ACS Chem. Biol.

Capella-Gutierrez, S., Silla-Martinez, J. M., and Gabaldon, T. (2009). trimAl: a tool for automated alignment trimming in large-scale phylogenetic analyses. Bioinformatics 25,

854 Casadevall, A. (2007). Determinants of virulence in the pathogenic fungi. Fungal Biol. Rev. 21, 130-132. doi:10.1016/j.fbr.2007.02.007.

Cock, P. J. A., Antao, T., Chang, J. T., Chapman, B. A., Cox, C. J., Dalke, A., et al. (2009). bioinformatics. Bioinformatics 25, 1422-1423. doi:10.1093/bioinformatics/btp163. Aspergillosis. Clin. Microbiol. Rev. 22, 447-465. doi:10.1128/CMR.00055-08.

Dolan, S. K., Owens, R. A., O’Keeffe, G., Hammel, S., Fitzpatrick, D. A., Jones, G. W., et al. 
Gliotoxin Biosynthesis in Aspergillus fumigatus. Chem. Biol. 21, 999-1012. doi:10.1016/j.chembiol.2014.07.006.

Drgona, L., Khachatryan, A., Stephens, J., Charbonneau, C., Kantecki, M., Haider, S., et al. (2014). Clinical and economic burden of invasive fungal diseases in Europe: focus on preemptive and empirical treatment of Aspergillus and Candida species. Eur. J. Clin. Microbiol. Infect. Dis. 33, 7-21. doi:10.1007/s10096-013-1944-3.

El-Elimat, T., Figueroa, M., Ehrmann, B. M., Cech, N. B., Pearce, C. J., and Oberlies, N. H. (2013). High-Resolution MS, MS/MS, and UV Database of Fungal Secondary Metabolites as a Dereplication Protocol for Bioactive Natural Products. J. Nat. Prod. 76, 1709-1716.

Emms, D. M., and Kelly, S. (2019). OrthoFinder: phylogenetic orthology inference for comparative genomics. Genome Biol. 20, 238. doi:10.1186/s13059-019-1832-y.

Fallon, J. P., Reeves, E. P., and Kavanagh, K. (2010). Inhibition of neutrophil function following exposure to the Aspergillus fumigatus toxin fumagillin. J. Med. Microbiol. 59, 625-633.

Fallon, J. P., Reeves, E. P., and Kavanagh, K. (2011). The Aspergillus fumigatus toxin 4. doi:10.1371/journal.pgen.1000046. discovery process. Nat. Prod. Rep. 32, 779-810. doi:10.1039/C4NP00134F. 
Gauthier, T., Wang, X., Sifuentes Dos Santos, J., Fysikopoulos, A., Tadrist, S., Canlet, C., et al. Cells. PLoS One 7, e29906. doi:10.1371/journal.pone.0029906.

889 González-Lobato, L., Real, R., Prieto, J. G., Álvarez, A. I., and Merino, G. (2010). Differential inhibition of murine Bcrp1/Abcg2 and human BCRP/ABCG2 by the mycotoxin fumitremorgin C. Eur. J. Pharmacol. 644, 41-48. doi:10.1016/j.ejphar.2010.07.016. Pathogenesis: To Air or Not To Air? Eukaryot. Cell 11, 560-570. doi:10.1128/EC.0003112.

Guruceaga, X., Ezpeleta, G., Mayayo, E., Sueiro-Olivares, M., Abad-Diaz-De-Cerio, A., Aguirre Urízar, J. M., et al. (2018). A possible role for fumagillin in cellular damage during host infection by Aspergillus fumigatus. Virulence 9, 1548-1561. doi:10.1080/21505594.2018.1526528.

Guruceaga, X., Perez-Cuesta, U., Abad-Diaz de Cerio, A., Gonzalez, O., Alonso, R. M., Hernando, F. L., et al. (2019). Fumagillin, a Mycotoxin of Aspergillus fumigatus: Biosynthesis, Biological Activities, Detection, and Applications. Toxins (Basel). 12, 7. doi:10.3390/toxins 12010007 .

Halász, J., Podányi, B., Vasvári-Debreczy, L., Szabó, A., Hajdú, F., Böcskei, Z., et al. (2000). 
Holt, C., and Yandell, M. (2011). MAKER2: an annotation pipeline and genome-database management tool for second-generation genome projects. BMC Bioinformatics 12, 491. doi:10.1186/1471-2105-12-491.

912 Houbraken, J., Weig, M., Groß, U., Meijer, M., and Bader, O. (2016). Aspergillus oerlinghausenensis, a new mould species closely related to A. fumigatus. FEMS Microbiol.

Hubert, J., Nuzillard, J.-M., and Renault, J.-H. (2017). Dereplication strategies in natural product research: How many tools and methodologies behind the same concept? Phytochem. Rev. 16, 55-95. doi:10.1007/s11101-015-9448-7.

921 Ito, T., and Masubuchi, M. (2014). Dereplication of microbial extracts and related analytical technologies. J. Antibiot. (Tokyo). 67, 353-360. doi:10.1038/ja.2014.12.

923 Jackson, R. W., Johnson, L. J., Clarke, S. R., and Arnold, D. L. (2011). Bacterial pathogen

924 evolution: breaking news. Trends Genet. 27, 32-40. doi:10.1016/j.tig.2010.10.001.

925 Kalyaanamoorthy, S., Minh, B. Q., Wong, T. K. F., von Haeseler, A., and Jermiin, L. S. (2017).

926 ModelFinder: fast model selection for accurate phylogenetic estimates. Nat. Methods 14, 587-589. doi:10.1038/nmeth.4285.

928 Kamei, K., and Watanabe, A. (2005). Aspergillus mycotoxins and their effect on the host. Med.

$929 \quad$ Mycol. 43, 95-99. doi:10.1080/13693780500051547.

930 Kato, N., Suzuki, H., Takagi, H., Asami, Y., Kakeya, H., Uramoto, M., et al. (2009).

931 Identification of Cytochrome P450s Required for Fumitremorgin Biosynthesis in 
Aspergillus fumigatus. ChemBioChem 10, 920-928. doi:10.1002/cbic.200800787.

933 Katoh, K., and Standley, D. M. (2013). MAFFT Multiple Sequence Alignment Software Version

934 7: Improvements in Performance and Usability. Mol. Biol. Evol. 30, 772-780.

935 doi:10.1093/molbev/mst010.

936 Kautsar, S. A., Blin, K., Shaw, S., Navarro-Muñoz, J. C., Terlouw, B. R., van der Hooft, J. J. J.,

937 et al. (2019). MIBiG 2.0: a repository for biosynthetic gene clusters of known function.

$938 \quad$ Nucleic Acids Res. doi:10.1093/nar/gkz882.

939 Keller, N. P. (2017). Heterogeneity confounds establishment of "a" model microbial strain. MBio

$940 \quad$ 8. doi:10.1128/mBio.00135-17.

941 Keller, N. P. (2019). Fungal secondary metabolism: regulation, function and drug discovery. Nat.

942 Rev. Microbiol. 17, 167-180. doi:10.1038/s41579-018-0121-1.

943 Khoufache, K., Puel, O., Loiseau, N., Delaforge, M., Rivollet, D., Coste, A., et al. (2007).

944 Verruculogen associated with Aspergillus fumigatus hyphae and conidia modifies the

945 electrophysiological properties of human nasal epithelial cells. BMC Microbiol. 7, 5.

946 doi:10.1186/1471-2180-7-5.

947 Knowles, S. L., Mead, M. E., Silva, L. P., Raja, H. A., Steenwyk, J. L., Goldman, G. H., et al.

948 (2020). Gliotoxin, a Known Virulence Factor in the Major Human Pathogen Aspergillus

949 fumigatus, Is Also Biosynthesized by Its Nonpathogenic Relative Aspergillus fischeri.

$950 \quad$ MBio 11. doi:10.1128/mBio.03361-19.

951 Knowles, S. L., Vu, N., Todd, D. A., Raja, H. A., Rokas, A., Zhang, Q., et al. (2019). Orthogonal

952 Method for Double-Bond Placement via Ozone-Induced Dissociation Mass Spectrometry

953 (OzID-MS). J. Nat. Prod. 82, 3421-3431. doi:10.1021/acs.jnatprod.9b00787.

954 Korf, I. (2004). Gene finding in novel genomes. BMC Bioinformatics 5, 59. doi:10.1186/1471- 
956 Kowalski, C. H., Beattie, S. R., Fuller, K. K., McGurk, E. A., Tang, Y.-W., Hohl, T. M., et al. (2016). Heterogeneity among Isolates Reveals that Fitness in Low Oxygen Correlates with Aspergillus fumigatus Virulence. MBio 7. doi:10.1128/mBio.01515-16.

Kowalski, C. H., Kerkaert, J. D., Liu, K.-W., Bond, M. C., Hartmann, R., Nadell, C. D., et al. (2019). Fungal biofilm morphology impacts hypoxia fitness and disease progression. Nat.

Kvalheim, O. M., Chan, H., Benzie, I. F. F., Szeto, Y., Tzang, A. H., Mok, D. K., et al. (2011). contributions from individual components to the bioactive signature in natural products. fumigatus, an endophytic fungus associated with Melia azedarach, and their antifungal, Alcaligenes faecalis A72 associated with South China Sea sponge Stelletta tenuis. Biochem.

974 Lind, A. L., Wisecaver, J. H., Lameiras, C., Wiemann, P., Palmer, J. M., Keller, N. P., et al.

975 (2017). Drivers of genetic diversity in secondary metabolic gene clusters within a fungal species. PLoS Biol. 15. doi:10.1371/journal.pbio.2003583.

977 Lind, A. L., Wisecaver, J. H., Smith, T. D., Feng, X., Calvo, A. M., and Rokas, A. (2015). 
Examining the evolution of the regulatory circuit controlling secondary metabolism and development in the fungal genus Aspergillus. PLoS Genet. 11, e1005096. doi:10.1371/journal.pgen.1005096.

Ma, Y. ., Li, Y., Liu, J. ., Song, Y. ., and Tan, R. . (2004). Anti-Helicobacter pylori metabolites from Rhizoctonia sp. Cy064, an endophytic fungus in Cynodon dactylon. Fitoterapia 75, 451-456. doi:10.1016/j.fitote.2004.03.007.

Mead, M. E., Knowles, S. L., Raja, H. A., Beattie, S. R., Kowalski, C. H., Steenwyk, J. L., et al. (2019a). Characterizing the Pathogenic, Genomic, and Chemical Traits of Aspergillus fischeri, a Close Relative of the Major Human Fungal Pathogen Aspergillus fumigatus.

Mead, M. E., Raja, H. A., Steenwyk, J. L., Knowles, S. L., Oberlies, N. H., and Rokas, A. (2019b). Draft Genome Sequence of the Griseofulvin-Producing Fungus Xylaria

Moran, G. P., Coleman, D. C., and Sullivan, D. J. (2011). Comparative Genomics and the Evolution of Pathogenicity in Human Pathogenic Fungi. Eukaryot. Cell 10, 34-42. doi:10.1128/EC.00242-10.

995

Navarro-Muñoz, J. C., Selem-Mojica, N., Mullowney, M. W., Kautsar, S. A., Tryon, J. H., Biol. Evol. 32, 268-274. doi:10.1093/molbev/msu300.

1000 Pluskal, T., Castillo, S., Villar-Briones, A., and Orešič, M. (2010). MZmine 2: Modular 
1001

1002

1003

1004

1005

1006

1007

1008

1009

1010

1011

1012

1013

1014

1015

1016

1017

1018

1019

1020

1021

1022

1023

framework for processing, visualizing, and analyzing mass spectrometry-based molecular profile data. BMC Bioinformatics 11, 395. doi:10.1186/1471-2105-11-395.

Poukka, H., Aarnisalo, P., Santti, H., Jänne, O. A., and Palvimo, J. J. (2000). Coregulator Small Nuclear RING Finger Protein (SNURF) Enhances Sp1- and Steroid Receptor-mediated Transcription by Different Mechanisms. J. Biol. Chem. 275, 571-579. doi:10.1074/jbc.275.1.571.

Raffa, N., and Keller, N. P. (2019). A call to arms: Mustering secondary metabolites for success and survival of an opportunistic pathogen. PLOS Pathog. 15, e1007606. doi:10.1371/journal.ppat.1007606.

Raftery, A. E., and Lewis, S. M. (1995). The number of iterations, convergence diagnostics and generic Metropolis algorithms. Pract. Markov Chain Monte Carlo 7, 763-773. doi:10.1.1.41.6352.

Ries, L. N. A., Steenwyk, J. L., de Castro, P. A., de Lima, P. B. A., Almeida, F., de Assis, L. J., et al. (2019). Nutritional Heterogeneity Among Aspergillus fumigatus Strains Has Consequences for Virulence in a Strain- and Host-Dependent Manner. Front. Microbiol. 10. doi:10.3389/fmicb.2019.00854.

Rokas, A., Mead, M. E., Steenwyk, J. L., Oberlies, N. H., and Goldman, G. H. (2020a). Evolving moldy murderers: Aspergillus section Fumigati as a model for studying the repeated evolution of fungal pathogenicity. PLOS Pathog. 16, e1008315. doi:10.1371/journal.ppat.1008315.

Rokas, A., Mead, M. E., Steenwyk, J. L., Raja, H. A., and Oberlies, N. H. (2020b). Biosynthetic gene clusters and the evolution of fungal chemodiversity. Nat. Prod. Rep. doi:10.1039/C9NP00045C. 
1024 Rokas, A., Williams, B. L., King, N., and Carroll, S. B. (2003). Genome-scale approaches to

1025 resolving incongruence in molecular phylogenies. Nature 425, 798-804.

1026 doi:10.1038/nature02053.

1027 Rokas, A., Wisecaver, J. H., and Lind, A. L. (2018). The birth, evolution and death of metabolic

1029 Samson, R. A., Hong, S., Peterson, S. W., Frisvad, J. C., and Varga, J. (2007). Polyphasic

1030 taxonomy of Aspergillus section Fumigati and its teleomorph Neosartorya. Stud. Mycol. 59,

Santos, R. A. C. dos, Steenwyk, J. L., Rivero-Menendez, O., Mead, M. E., Silva, L. P., Bastos, 147-203. doi:10.3114/sim.2007.59.14. R. W., et al. (2020). Genomic and phenotypic heterogeneity of clinical isolates of the

Stanke, M., and Waack, S. (2003). Gene prediction with a hidden Markov model and a new human pathogens Aspergillus fumigatus, Aspergillus lentulus and Aspergillus intron submodel. Bioinformatics 19, ii215-ii225. doi:10.1093/bioinformatics/btg1080.

Steenwyk, J. L., Shen, X.-X., Lind, A. L., Goldman, G. H., and Rokas, A. (2019). A Robust Phylogenomic Time Tree for Biotechnologically and Medically Important Fungi in the

Steenwyk, J., and Rokas, A. (2017). Extensive Copy Number Variation in Fermentation-Related Genes Among Saccharomyces cerevisiae Wine Strains. G3 Genes, Genomes, Genet. 7.

1044 Sugui, J. A., Pardo, J., Chang, Y. C., Zarember, K. A., Nardone, G., Galvez, E. M., et al. (2007). 1045 Gliotoxin Is a Virulence Factor of Aspergillus fumigatus : gliP Deletion Attenuates 1046 Virulence in Mice Immunosuppressed with Hydrocortisone. Eukaryot. Cell 6, 1562-1569. 
doi:10.1128/EC.00141-07.

1048 Tavaré, S. (1986). Some probabilistic and statistical problems in the analysis of DNA sequences.

1049 Lect. Math. life Sci. 17, 57-86.

1050 Tekaia, F., and Latgé, J.-P. (2005). Aspergillus fumigatus: saprophyte or pathogen? Curr. Opin.

1051 Microbiol. 8, 385-392. doi:10.1016/j.mib.2005.06.017.

1052 Vallabhaneni, S., Mody, R. K., Walker, T., and Chiller, T. (2016). The Global Burden of Fungal

1053 Diseases. Infect. Dis. Clin. North Am. 30, 1-11. doi:10.1016/j.idc.2015.10.004.

1054 van Dongen, S. (2000). Graph clustering by flow simulation. Graph Stimul. by flow Clust. PhD

1055 thesis, University of Utrecht. doi:10.1016/j.cosrev.2007.05.001.

1056 Vinet, L., and Zhedanov, A. (2011). A 'missing' family of classical orthogonal polynomials. $J$.

1057 Phys. A Math. Theor. 44, 085201. doi:10.1088/1751-8113/44/8/085201.

1058 Wang, F.-Z., Li, D.-H., Zhu, T.-J., Zhang, M., and Gu, Q.-Q. (2011). Pseurotin A 1 and A 2 , two

1059 new 1-oxa-7-azaspiro[4.4]non-2-ene-4,6-diones from the holothurian-derived fungus

1060 Aspergillus fumigatus WFZ-25. Can. J. Chem. 89, 72-76. doi:10.1139/V10-157.

1061 Wang, F., Fang, Y., Zhu, T., Zhang, M., Lin, A., Gu, Q., et al. (2008). Seven new prenylated

1062 indole diketopiperazine alkaloids from holothurian-derived fungus Aspergillus fumigatus.

1063 Tetrahedron 64, 7986-7991. doi:10.1016/j.tet.2008.06.013.

1064 Waterhouse, R. M., Seppey, M., Simão, F. A., Manni, M., Ioannidis, P., Klioutchnikov, G., et al.

1065 (2018). BUSCO Applications from Quality Assessments to Gene Prediction and

1066 Phylogenomics. Mol. Biol. Evol. 35, 543-548. doi:10.1093/molbev/msx319.

1067 Waterhouse, R. M., Tegenfeldt, F., Li, J., Zdobnov, E. M., and Kriventseva, E. V. (2013).

1068 OrthoDB: a hierarchical catalog of animal, fungal and bacterial orthologs. Nucleic Acids

1069 Res. 41, D358-D365. doi:10.1093/nar/gks1116. 
1070 Weber, T., Blin, K., Duddela, S., Krug, D., Kim, H. U., Bruccoleri, R., et al. (2015). antiSMASH

1071

1072

1073

1074

1075

1076

1077

1078

1079

1080

1081

1082

1083

1084

1085

1086

1087

1088

1089

1090

1091

1092

3.0 - a comprehensive resource for the genome mining of biosynthetic gene clusters.

Nucleic Acids Res. 43, W237-W243. doi:10.1093/nar/gkv437.

Wiemann, P., Guo, C.-J., Palmer, J. M., Sekonyela, R., Wang, C. C. C., and Keller, N. P. (2013).

Prototype of an intertwined secondary-metabolite supercluster. Proc. Natl. Acad. Sci. 110, 17065-17070. doi:10.1073/pnas.1313258110.

Yamada, A., Kataoka, T., and Nagai, K. (2000). The fungal metabolite gliotoxin:

immunosuppressive activity on CTL-mediated cytotoxicity. Immunol. Lett. 71, 27-32.

doi:10.1016/s0165-2478(99)00155-8.

Yandell, M., and Ence, D. (2012). A beginner's guide to eukaryotic genome annotation. Nat.

Rev. Genet. 13, 329-42. doi:10.1038/nrg3174.

Yang, Z. (1994). Maximum likelihood phylogenetic estimation from DNA sequences with variable rates over sites: Approximate methods. J. Mol. Evol. 39, 306-314. doi:10.1007/BF00160154.

Yang, Z. (1996). Among-site rate variation and its impact on phylogenetic analyses. Trends Ecol. Evol. 11, 367-372. doi:10.1016/0169-5347(96)10041-0.

Yang, Z. (2007). PAML 4: Phylogenetic Analysis by Maximum Likelihood. Mol. Biol. Evol. 24, 1586-1591. doi:10.1093/molbev/msm088.

Zhao, J., Mou, Y., Shan, T., Li, Y., Zhou, L., Wang, M., et al. (2010). Antimicrobial Metabolites from the Endophytic Fungus Pichia guilliermondii Isolated from Paris polyphylla var. yunnanensis. Molecules 15, 7961-7970. doi:10.3390/molecules15117961.

Zhao, S., Latgé, J.-P., and Gibbons, J. G. (2019). Genome Sequences of Two Strains of the Food Spoilage Mold Aspergillus fischeri. Microbiol. Resour. Announc. 8. 
bioRxiv preprint doi: https://doi.org/10.1101/2020.04.09.033902; this version posted April 10, 2020. The copyright holder for this preprint (which was not certified by peer review) is the author/funder, who has granted bioRxiv a license to display the preprint in perpetuity. It is made available under aCC-BY-NC 4.0 International license.

1093 doi:10.1128/MRA.01328-19.

1094 